\title{
Exponential Synchronization of Complex Delayed Dynamical Networks With Switching Topology
}

\author{
Tao Liu, Jun Zhao, and David J. Hill, Fellow, IEEE
}

\begin{abstract}
This paper studies the local and global exponential synchronization of a complex dynamical network with switching topology and time-varying coupling delays. By using stability theory of switched systems and the network topology, the synchronization of such a network under some special switching signals is investigated. Firstly, under the assumption that all subnetworks are self-synchronizing, a delay-dependent sufficient condition is given in terms of linear matrix inequalities, which guarantees the solvability of the local synchronization problem under an average dwell time scheme. Then this result is extended to the situation that not all subnetworks are self-synchronizing. For the latter case, in addition to average dwell time, an extra condition on the ratio of the total activation time of self-synchronizing and nonsynchronizing subnetworks is needed to achieve synchronization of the entire switched network. The global synchronization of a network whose isolate dynamics is of a particular form is also studied. Three different examples of delayed dynamical networks with switching topology are given, which demonstrate the effectiveness of obtained results.
\end{abstract}

Index Terms-Average dwell time, complex dynamical network, exponential synchronization, switched systems, switching topology, time-varying delays.

\section{INTRODUCTION}

$\mathbf{M}$ OTIVATED by many systems in science and humanities, complex dynamical networks which consist of interacting dynamical entities with an interplay between dynamical states and interaction patterns have been extensively studied in past decades [1]-[3]. Many systems in nature can be modeled by complex dynamical networks, for example, power grids, communication networks, Internet, the World Wide Web, metabolic systems, food webs, etc.

Synchronization, as an important collective behavior of complex dynamical networks, has received much of the focus. Early works on this issue concentrated on networks with regular topological structures to capture complexities caused by the nonlinear dynamics of the nodes [4], [5]. Inspired by the seminal work [6], attention has been moved to complexities coming from network structures. Sufficient conditions on synchronization of various kinds of networks with time-varying

Manuscript received October 03, 2009; revised April 02, 2010; accepted May 10, 2010. Date of publication July 23, 2010; date of current version November 10, 2010. This work was supported by the Australian Research Council's Discovery Projects FF 0455875. This paper was recommended by Associate Editor M. Di Marco.

T. Liu and J. Zhao are with the School of Engineering, Australian National University, Canberra ACT 0200, Australia (e-mail: tao.liu@anu.edu.au; jun. zhao@anu.edu.au).

D. J. Hill is with the School of Engineering, Australian National University, Canberra ACT 0200, Australia. He is also with National ICT Australia, Canberra, ACT 0200, Australia (e-mail: david.hill@anu.edu.au).

Digital Object Identifier 10.1109/TCSI.2010.2052508 couplings [7]-[9], time delay couplings [10]-[14], directed and weighted couplings [15]-[17], and the relationship between the synchronizability and the properties of network structures [18]-[20] have been investigated - to name just a few; and see a recent survey and monograph [21], [22] for more details.

In the past decade, synchronization of networks with switching topology has attracted researchers' interest [24]-[37]. Switching topology often due to link failures or new creation is general in real-world networks. Take a power grid as an example. It is well known that a large power grid consisting of a large number of local power generators can work properly only if the generators are kept in synchronism, and needs to retain its stability to provide normal power supplies to consumers. However, loss of synchronization of some local power generators may result in instability of the power grid, or even cause cascading outages or collapse and blackout [47], [48]. So, when a local power system happens to have a severe fault, it will be automatically cut off from the network by a relay protection device to avoid further damage to the global power grid. This makes the connection structure jump suddenly-switch from one topology to another. On the other hand, new links between a certain group of local power generators may be established for certain purposes such as preventing cascading instability [48]. Switching topology also happens in the communication network between mobile agents (the nodes) which communicate with each other and need to perform synchronization. Since the nodes of the network are moving, some existing links can fail and new links between nearby agents are created [23].

Inherently, switching topology is discontinuously "fastvarying" topology and in general cannot be handled as general time-varying topology. Several methods have been proposed to deal with switching topology in special network studies. In [24], a blinking network in which the coupling is switched according to certain probability was addressed. Such a network was shown to synchronize for almost all instances of the fast switching random process. It was shown in [25] that if the switching is fast enough, then an average model can be applied with certain properties of the original network preserved, and local synchronization is achieved if the static time-average of the network supports synchronization. This result was extended to the stochastic network in [29] and [36]. In order to synchronize a network, [35] also applied this idea to pinning control strategy by switching a single pinning controller sufficient fast from one node to another. The differences of synchronization properties between the switched network and corresponding time-average network were investigated in [31]. In [34], the sychronizability of discrete-time networks was measured by Hajnal diameter of infinite coupling matrices, and different network models including random switching between the individual topologies 
were discussed in [33]. Numerical studies of a network whose coupling strength switched on and off periodically were carried out in [32]. A similar idea was used to synchronize the network by periodically intermittent pinning control, and corresponding synchronization criteria were obtained in [37]. An adaptive controller was designed to synchronize a switched network under arbitrary switching in [26]. The authors in [27] investigated the synchronization of switched networks whose outer coupling matrices are simultaneously triangularized under arbitrary switching as well as via design of switching within a pregiven collection of topologies in which synchronization cannot be achieved by using any topology alone. Another class of problems which deal with switched networks is in consensus control [23], [44], [49]. All of these have advanced the understanding of the synchronization phenomenon in real-world networks with switching topology.

From a control point of view, the fast switching property may not always desirable because it will probably shorten the working life of actuating mechanism, and sometimes fast switching is not allowed in some real-world networks such as power grid. Moreover, the switched system theory shows that the stability of a switched system can not be guaranteed even when switching happens between two exponentially stable subsystems, and one way to preserve stability of the system is to use the so-called slow switching law [38]. These stimulate the studies on the synchronizability of switched networks where the switching signal belongs to "slow" switching laws, i.e., how to identify those switching signals which guarantee the synchronization of switched network. Some recent progress in this direction has been made in [28] and [30] by using the averaged dwell time method [39]-[42]. In [28], local synchronization of a network with unweighed switching coupling matrices was investigated, and a criterion on how to determine its coupling strength to preserve synchronization under a specified switching law was given. Synchronization criteria of a switched network with time-invariant coupling delay were given under an assumption that all outer coupling matrices are simultaneously diagonalizable in [30].

In addition, another problem that deserves attention is the synchronization of networks with time-varying coupling delays. Time delay is a ubiquitous phenomenon in networks due to the limited speed of signals traveling through links. Thus, there often exist coupling delays in networks, and the existence of time delay makes the dynamical behaviors of a network much more complicated. So it is also meaningful to consider the effect caused by time delay to networks. Although numerous studies [10]-[13] have considered delayed networks, the case of timevarying delay remains to be fully studied.

In this paper, we investigate the local and global exponential synchronization of a class of dynamical networks with switching topology and time-varying delays whose inner and outer coupling matrices take values in two finite sets of matrices via a switching signal, and we do not require simultaneously diagonalizable/triangularizable condition on the outer coupling matrices, which is quite restrictive in practice. Firstly, by using the average dwell time method, we discuss the problem in two different cases: i) all subnetworks are self-synchronizing and ii) not all subnetworks are self-synchronizing. For both cases, we obtain corresponding linear matrix inequality
(LMI)-based delay-dependent sufficient conditions and identify the switching signals for local exponential synchronization of the overall network. The conditions are completely decoupled lower dimensional LMIs, which can be applied to large scale networks directly. Then, we specify a network model which has a particular isolated node dynamics and study the global exponential synchronization of such a network.

The rest of this paper is organized as follows. We first introduce a dynamical network model with switching topology and time-varying coupling delays in Section II. This is followed by our main results in Section III and Section IV where we give the theoretical analysis of local and global exponential synchronization for such a network in the cases of all and some subnetworks being self-synchronizing. In Section V, examples with different subnetworks are given to demonstrate the effectiveness of the theoretical results. Finally, conclusions are presented in Section VI.

\section{Model DescriPtion AND PRELIMINARIES}

To represent topology changes of some real-world networks, we introduce a new model for a network with switching topology and time-varying coupling delays. The network consists of $N$ linearly and diffusively coupled identical nodes with each node being an $n$-dimensional dynamical system. The interconnections between nodes are changed abruptly at some time instant which is orchestrated by a switching signal. The state equations of the switched network are given below

$$
\begin{aligned}
\dot{x}_{i}(t) & =f\left(x_{i}(t)\right)+\sum_{j=1}^{N} c_{i j}^{\sigma(t)} \Gamma_{\sigma(t)} x_{j}(t-d(t)) \\
x_{i t_{0}} & =\varphi_{i}(\theta), \quad \theta \in[-\tau, 0], \quad i \in \mathcal{N}
\end{aligned}
$$

where $x_{i}=\left(x_{i 1}, x_{i 2}, \ldots, x_{i n}\right)^{\top} \in \mathbb{R}^{n}, \forall i \in \mathcal{N}=$ $\{1,2, \ldots, N\}$ are the state variables of node $i$, and $d(t) \geq 0$ is time-varying delay; $\varphi_{i}(\theta)$ are continuously differentiable initial functions, $\tau>0$ is a known constant and $x_{i t}=x_{i}(t+\theta)$, $\theta \in[-\tau, 0] ; f(\cdot): \mathbb{R}^{n} \rightarrow \mathbb{R}^{n}$ is continuously differentiable; $\sigma(t):[0, \infty) \rightarrow \mathcal{M}=\{1,2, \ldots, m\}$ is the switching signal, which is a piecewise constant function continuous from the right, i.e., it is constant between two consecutive discontinuities; for each fixed $\sigma(t)=k \in \mathcal{M}, \Gamma_{k} \in \mathbb{R}^{n \times n}$ is the inner coupling matrix which describes the way of linking the components in each pair of connected two nodes; $C_{k} \in \mathbb{R}^{N \times N}$ is the outer coupling configuration matrix representing the coupling strength and topology of the network; if there is a connection between node $i$ and node $j(i \neq j)$, then $c_{i j}^{k}=c_{j i}^{k}>0$; otherwise $c_{i j}^{k}=c_{j i}^{k}=0$, and the diagonal elements of matrix $C_{k}$ satisfy

$$
c_{i i}^{k}=-\sum_{\substack{j=1 \\ j \neq i}}^{N} c_{i j}^{k}=-\sum_{\substack{j=1 \\ j \neq i}}^{N} c_{j i}^{k}, \quad i \in \mathcal{N}, \quad k \in \mathcal{M} .
$$

Actually, the delayed dynamical network (1) with switching topology is generated by a switching signal $\sigma(t)$ and a family of subnetworks (3) which have the same isolate dynamics $f(\cdot)$, but different inner and outer coupling matrices $\Gamma_{k}$ and $C_{k}$

$$
\begin{aligned}
\dot{x}_{i}(t) & =f\left(x_{i}(t)\right)+\sum_{j=1}^{N} c_{i j}^{k} \Gamma_{k} x_{j}(t-d(t)), \quad k \in \mathcal{M} \\
x_{i t_{0}} & =\varphi_{i}(\theta), \quad \theta \in[-\tau, 0] .
\end{aligned}
$$


The switching signal $\sigma(t)$ specifies at every time the index of the active subnetworks $k \in \mathcal{M}$. We assume that the switching signal $\sigma(t)$ has a finite number of discontinuities in any finite time interval, and there are no jumps in the state at the switching instants.

Corresponding to the switching signal $\sigma(t)$, we have the switching sequence $\left\{x_{t_{0}} ;\left(k_{0}, t_{0}\right), \ldots,\left(k_{r}, t_{r}\right), \ldots, \mid k_{r} \quad \in\right.$ $\mathcal{M}, r=0,1, \ldots\}$, which means that the $k_{r}$ th subnetwork is activated when $t \in\left[t_{r}, t_{r+1}\right)$.

Remark 1: For a fixed $\sigma(t)$, the network (1) without time delays appears to be the form of the time-varying network proposed in [7]. However, there are important differences: the timevarying network admits a family of solutions that can be parameterized solely by the initial condition; however, the network with switching topology admits a family of solutions that are parameterized both by the initial condition and the switching signal $\sigma(t)$. Also, in a time-varying network, the coupling matrix is continuous and often differentiable with respect to time $t$, where $\sigma(t)$ is usually discontinuous but piecewise constant. Therefore, typically theories and methods of time-varying networks can not be applied to networks with switching topology.

Our task in this paper is to find conditions which guarantee the local and global exponential synchronization of the states of the network (1) on the manifold defined below.

Definition 1 ([11]): Define synchronization manifold $\mathbb{S}=$ $\left\{\left(x_{1}^{\top}, x_{2}^{\top}, \ldots, x_{N}^{\top}\right)^{\top} \in \mathbb{R}^{n N}: x_{i}=x_{j} i, j \in \mathcal{N}\right\}$.

Let $s(t)=(1 / N) \sum_{i=1}^{N} x_{i}(t)$, then the dynamical equation of $s(t)$ can be written as

$$
\dot{s}(t)=\frac{1}{N} \sum_{i=1}^{N} \dot{x}_{i}(t)=\frac{1}{N} \sum_{i=1}^{N} f\left(x_{i}(t)\right) .
$$

Obviously, the stability of the synchronous manifold of the switched network (1) is determined by the dynamics of the isolate node $f(\cdot)$, the inner coupling matrices $\Gamma_{k}$, the outer coupling matrices $C_{k}$, the time-varying delay $d(t)$ and the switching signal $\sigma(t)$. It turns out that not all the switching signals exhibit sufficient regularity for our purpose, so we specify the switching signal with some "better-behaved" property in this paper, i.e., average dwell time, which is defined as below.

Definition 2 ([38], [39]): For any $T_{2}>T_{1} \geq 0$, let $N_{\sigma}\left(T_{1}, T_{2}\right)$ denote the number of switching of $\sigma(t)$ over $\left(T_{1}, T_{2}\right)$. If $N_{\sigma}\left(T_{1}, T_{2}\right) \leq N_{0}+\left(T_{2}-T_{1}\right) / T_{a}$ holds for $T_{a}>0, N_{0} \geq 0$, then $T_{a}$ is called average dwell time.

From Definition 2, we see that in any open interval, the number of discontinuities of $\sigma(t)$ is bounded above by the length of the interval normalized by $T_{a}$ plus a "chatter bound" $N_{0}$ [42]. So arbitrarily fast switching is not allowed, which is natural in practice.

In the following, we give two assumptions on time-varying delay $d(t)$ and a useful lemma.

Assumption $1([40]): d(t)$ is a differentiable function satisfying $0 \leq d(t) \leq \tau$ and $\dot{d}(t) \leq d<1$ for some known constant $d$.

Assumption 2([40]): $d(t)$ is a continuous function satisfying $0 \leq d(t) \leq \tau$.

Noting that Assumption 2 is a delay-derivative-free condition, it means that the derivative of $d(t)$ can be unknown or arbitrary.

Lemma 1 ([43]): The linear matrix inequality

$$
\left(\begin{array}{ll}
Q & S \\
* & R
\end{array}\right)>0
$$

with $Q=Q^{\top}, R=R^{\top}$ and $*$ referring to the symmetric terms in a symmetric matrix, is equivalent to

$$
R>0, \quad Q-S R^{-1} S^{\top}>0 .
$$

\section{LOCAL EXPONENTIAL SYNCHRONIZATION}

In order to achieve the objective at the manifold $\mathbb{S}$, we define the error vector

$$
e_{i}(t)=x_{i}(t)-s(t), \quad i \in \mathcal{N} .
$$

Subtracting (4) from (1) yields the error dynamical systems

$$
\begin{aligned}
\dot{e}_{i}(t)=f\left(e_{i}(t)\right. & +s(t))-\frac{1}{N} \sum_{p=1}^{N} f\left(e_{p}(t)+s(t)\right) \\
& +\sum_{j=1}^{N} c_{i j}^{\sigma(t)} \Gamma_{\sigma(t)} e_{j}(t-d(t)), \quad i \in \mathcal{N} .
\end{aligned}
$$

Then the exponential synchronization problem of the dynamical network (1) is equivalent to the problem of exponential stability of the error dynamical systems (6). Since $f(\cdot)$ is continuously differentiable, local exponential synchronization can be assessed by examining exponential stability of the following linear switched delay systems:

$$
\dot{e}_{i}(t)=A(t) e_{i}(t)+\sum_{j=1}^{N} c_{i j}^{\sigma(t)} \Gamma_{\sigma(t)} e_{j}(t-d(t)), \quad i \in \mathcal{N}
$$

where $A(t)=D f(s(t))$ is the Jacobian of $f(s(t))$ on $s(t)$.

Let $e(t)=\left(e_{1}^{\top}(t), e_{2}^{\top}(t), \ldots, e_{N}^{\top}(t)\right)^{\top} \in \mathbb{R}^{n N}$, we can rewrite (7) as

$$
\dot{e}(t)=\left(I_{N} \otimes A(t)\right) e(t)+\left(C_{\sigma(t)} \otimes \Gamma_{\sigma(t)}\right) e(t-d(t))
$$

where $I_{N} \in \mathbb{R}^{N \times N}$ is the identity matrix, and $\otimes$ is the Kronecker product of matrices.

For each $k \in \mathcal{M}$, we have

$$
\dot{e}(t)=\bar{A}(t) e(t)+\bar{C}_{k} e(t-d(t))
$$

with $\bar{A}(t)=I_{N} \otimes A(t)$ and $\bar{C}_{k}=C_{k} \otimes \Gamma_{k}$.

Since $C_{k}=C_{k}^{\top}$ is a real symmetric matrix, there exists a unitary matrix $U_{k}=\left(u_{k 1}, u_{k 2}, \ldots, u_{k N}\right) \in \mathbb{R}^{N \times N}$ with $u_{k i}=$ $\left(u_{1 i}^{k}, u_{2 i}^{k}, \ldots, u_{N i}^{k}\right)^{\top} \in \mathbb{R}^{N}$ such that

$$
U_{k}^{\top} C_{k} U_{k}=\Lambda_{k}
$$

where $U_{k}^{\top} U_{k}=I_{N}, \Lambda_{k}=\operatorname{diag}\left\{\lambda_{k 1}, \lambda_{k 2}, \ldots, \lambda_{k N}\right\}, \lambda_{k i}, i \in$ $\mathcal{N}$ are the eigenvalues of $C_{k}$. The zero row sum condition (2) ensures that: i) $\lambda_{k 1} \equiv 0$ with associated eigenvector $u_{k 1}=$ $1 / \sqrt{N}(1,1, \ldots, 1)^{\top}$ and ii) $0=\lambda_{k 1} \geq \lambda_{k 2} \geq \ldots \geq \lambda_{k N}$.

Using the unitary transform $y_{k}(t)=\left(\bar{U}_{k}^{\top} \otimes I_{n}\right) e(t)=$ $\left(y_{k 1}^{\top}(t), y_{k 2}^{\top}(t), \ldots, y_{k N}^{\top}(t)\right)^{\top} \in \mathbb{R}^{n N}$, along with (9), gives

$$
\dot{y}_{k}(t)=\bar{A}(t) y_{k}(t)+\bar{\Lambda}_{k} y_{k}(t-d(t))
$$

with $\bar{\Lambda}_{k}=\Lambda_{k} \otimes \Gamma_{k}$, i.e.,

$$
\dot{y}_{k i}(t)=A(t) y_{k i}(t)+\lambda_{k i} \Gamma_{k} y_{k i}(t-d(t)), \quad i \in \mathcal{N}, \quad k \in \mathcal{M}
$$

where $y_{k 1}(t)=\left(u_{k 1}^{\top} \otimes I_{n}\right) e(t)=(1 / \sqrt{N}) \sum_{i=1}^{N} e_{i}(t) \equiv \mathbf{0} \in$ $\mathbb{R}^{n}$. 
Next, we will find conditions on the dynamics of the isolate node $f(\cdot)$, the inner coupling matrices $\Gamma_{k}$, the outer coupling matrices $C_{k}$, the time-varying delay $d(t)$, and the switching signal $\sigma(t)$ under which local exponential synchronization of the network (1) is guaranteed for both cases of all and some subnetworks in (3) being self-synchronizing.

\section{A. All Subnetworks Are Self-Synchronizing}

In this subsection, we assume that all subnetworks in (3) are self-synchronizing. We analyze the exponential synchronization of switched network (1) when the subnetworks are switched by an average dwell time scheme.

Theorem 1: For a given constant $\alpha>0$, suppose Assumption 1 holds and there exist matrices $P_{k i}>0, Q_{k i}>0, Z_{k i}>0$, $Y_{k i}, T_{k i}$ and

$$
X_{k i}=\left(\begin{array}{cc}
X_{11}^{k i} & X_{12}^{k i} \\
* & X_{22}^{k i}
\end{array}\right) \geq 0
$$

with appropriate dimensions such that

$$
\begin{aligned}
\Phi_{k i}= & \left(\begin{array}{ccc}
\phi_{11}^{k i} & \phi_{12}^{k i} & \tau A^{\top}(t) Z_{k i} \\
* & \phi_{22}^{k i} & \tau \lambda_{k i} \Gamma_{k}^{\top} Z_{k i} \\
* & * & -\tau Z_{k i}
\end{array}\right)<0, \\
& i \in \mathcal{N}_{1}, \quad k \in \mathcal{M}, \\
\Theta_{k i}= & \left(\begin{array}{ccc}
X_{11}^{k i} & X_{12}^{k i} & Y_{k i} \\
* & X_{22}^{k i} & T_{k i} \\
* & * & \mathrm{e}^{-\alpha \tau} Z_{k i}
\end{array}\right) \geq 0, \\
& i \in \mathcal{N}_{1}, \quad k \in \mathcal{M} .
\end{aligned}
$$

Then the synchronous manifold $\mathbb{S}$ of the delayed network (1) is locally exponentially stable for any switching signal with average dwell time satisfying

$$
T_{a}>T_{a}^{*}=\frac{\ln \mu}{\alpha}
$$

where

$$
\begin{aligned}
\phi_{11}^{k i}= & P_{k i} A(t)+A^{\top}(t) P_{k i}+Y_{k i}^{\top}+Y_{k i}+Q_{k i}+\tau X_{11}^{k i} \\
& +\alpha P_{k i}, \\
\phi_{12}^{k i}= & \lambda_{k i} P_{k i} \Gamma_{k}-Y_{k i}+T_{k i}^{\top}+\tau X_{12}^{k i}, \\
\phi_{22}^{k i}= & -T_{k i}-T_{k i}^{\top}-(1-d) \mathrm{e}^{-\alpha \tau} Q_{k i}+\tau X_{22}^{k i}
\end{aligned}
$$

and $\mu \geq 1$ satisfies

$$
\tilde{P}_{k} \leq \mu \tilde{P}_{l}, \quad \tilde{Q}_{k} \leq \mu \tilde{Q}_{l}, \quad \tilde{Z}_{k} \leq \mu \tilde{Z}_{l}, \quad \forall k, l \in \mathcal{M}
$$

with $\quad \tilde{P}_{k}=\left(U_{k} \otimes I_{n}\right) \bar{P}_{k}\left(U_{k}^{\top} \otimes I_{n}\right), \quad \tilde{Q}_{k}=$ $\left(U_{k} \otimes I_{n}\right) \bar{Q}_{k}\left(U_{k}^{\top} \otimes I_{n}\right), \tilde{Z}_{k}=\left(U_{k} \otimes I_{n}\right) \bar{Z}_{k}\left(U_{k}^{\top} \otimes I_{n}\right) ;$ $\bar{P}_{k}=\operatorname{diag}\left\{P_{k 1}, P_{k 2}, \ldots, P_{k N}\right\} \in \mathbb{R}^{n N \times n N}$, $\bar{Q}_{k}=\operatorname{diag}\left\{Q_{k 1}, Q_{k 2}, \ldots, Q_{k N}\right\} \in \mathbb{R}^{n N \times n N}$, $\bar{Z}_{k}=\operatorname{diag}\left\{Z_{k 1}, Z_{k 2}, \ldots, Z_{k N}\right\} \in \mathbb{R}^{n N \times n N} . P_{k 1} \in \mathbb{R}^{n \times n}$, $Q_{k 1} \in \mathbb{R}^{n \times n}, Z_{k 1} \in \mathbb{R}^{n \times n}$ are arbitrary positive definite matrices, and $P_{k i} \in \mathbb{R}^{n \times n}, Q_{k i} \in \mathbb{R}^{n \times n}, Z_{k i} \in \mathbb{R}^{n \times n}$, $i \in \mathcal{N}_{1}=\{2,3, \ldots, N\}$ are the solutions of inequality (13) and (14).

Proof: Select the following piecewise Lyapunov functional candidate

$$
V(t)=V_{\sigma(t)}(t)=V_{1 \sigma(t)}+V_{2 \sigma(t)}+V_{3 \sigma(t)}
$$

where

$$
\begin{aligned}
& V_{1 \sigma(t)}=e^{\top}(t) \tilde{P}_{\sigma(t)} e(t), \\
& V_{2 \sigma(t)}=\int_{-\tau}^{0} \int_{t+\theta}^{t} \mathrm{e}^{\alpha(\beta-t)} \dot{e}^{\top}(\beta) \tilde{Z}_{\sigma(t)} \dot{e}(\beta) \mathrm{d} \beta \mathrm{d} \theta, \\
& V_{3 \sigma(t)}=\int_{t-d(t)}^{t} \mathrm{e}^{\alpha(\beta-t)} e^{\top}(\beta) \tilde{Q}_{\sigma(t)} e(\beta) \mathrm{d} \beta .
\end{aligned}
$$

On each interval $\left[t_{r}, t_{r+1}\right)$, the switching signal is constant, and for simplicity, let $\sigma(t)=k$ for $t \in\left[t_{r}, t_{r+1}\right)$. Obviously, $\forall t \in$ $\left[t_{r}, t_{r+1}\right)$ we have

$$
\begin{aligned}
\dot{V}_{1 k}= & \dot{e}^{\top}(t) \tilde{P}_{k} e(t)+e^{\top}(t) \tilde{P}_{k} \dot{e}(t) \\
= & \dot{y}_{k}^{\top}(t) \bar{P}_{k} y_{k}(t)+y_{k}^{\top}(t) \bar{P}_{k} \dot{y}_{k}(t) \\
= & \sum_{i=1}^{N}\left[y_{k i}^{\top}(t)\left(A^{\top}(t) P_{k i}+P_{k i} A(t)\right) y_{k i}(t)\right. \\
& \left.+2 y_{k i}^{\top}(t) P_{k i} \lambda_{k i} \Gamma_{k} y_{k i}(t-d(t))\right] \\
\dot{V}_{2 k} \leq & -\alpha V_{2 k}+\tau \dot{y}_{k}^{\top}(t) \bar{Z}_{k} \dot{y}_{k}(t) \\
& -\int_{t-d(t)}^{t} \mathrm{e}^{-\alpha \tau} \dot{y}_{k}^{\top}(\beta) \bar{Z}_{k} \dot{y}_{k}(\beta) \mathrm{d} \beta \\
= & -\alpha V_{2 k} \\
& +\sum_{i=1}^{N}\left[\tau \dot{y}_{k i}^{\top}(t) Z_{k i} \dot{y}_{k i}(t)\right. \\
& \left.\quad \int_{t-d(t)}^{t} \mathrm{e}^{-\alpha \tau} \dot{y}_{k i}^{\top}(\beta) Z_{k i} \dot{y}_{k i}(\beta) \mathrm{d} \beta\right], \\
\dot{V}_{3 k} \leq & -\alpha V_{3 k}+y_{k}^{\top}(t) \bar{Q}_{k} y_{k}(t)-(1-d) \mathrm{e}^{-\alpha \tau} \\
& \times y_{k}^{\top}(t-d(t)) \bar{Q}_{k} y_{k}(t-d(t)) \\
= & -\alpha V_{3 k} \quad+\sum_{i=1}^{N}\left[y_{k i}^{\top}(t) Q_{k i} y_{k i}(t)-(1-d) \mathrm{e}^{-\alpha \tau}\right. \\
& \left.\times y_{k i}^{\top}(t-d(t)) Q_{k i} y_{k i}(t-d(t))\right] .
\end{aligned}
$$

By the Leibniz-Newton formula, we have

$$
\int_{t-d(t)}^{t} \dot{y}_{k i}(\beta) \mathrm{d} \beta=y_{k i}(t)-y_{k i}(t-d(t)) .
$$

Then for any matrices $Y_{k i}$ and $T_{k i}$ with appropriate dimensions, we get

$$
\begin{aligned}
& 2\left[y_{k i}^{\top}(t) Y_{k i}+y_{k i}^{\top}(t-d(t)) T_{k i}\right] \times \\
& {\left[y_{k i}(t)-\int_{t-d(t)}^{t} \dot{y}_{k i}(\beta) \mathrm{d} \beta-y_{k i}(t-d(t))\right]=0 .}
\end{aligned}
$$

Obviously, for any matrix $X_{k i} \geq 0$, it holds that

$$
\tau \varpi_{k i}^{\top}(t) X_{k i} \varpi_{k i}(t) \geq \int_{t-d(t)}^{t} \varpi_{k i}^{\top}(t) X_{k i} \varpi_{k i}(t) \mathrm{d} \beta,
$$


where $\varpi_{k i}(t)=\left(y_{k i}^{\top}(t), y_{k i}^{\top}(t-d(t))\right)^{\top}$.

Combining (17)-(23) and with $y_{k 1} \equiv \mathbf{0} \in \mathbb{R}^{n}$ lead to

$$
\begin{aligned}
\dot{V}(t)+\alpha V(t) \leq \sum_{i=2}^{N} & {\left[\varpi_{k i}^{\top}(t) \hat{\Phi}_{k i} \varpi_{k i}(t)\right.} \\
& \left.-\int_{t-d(t)}^{t} \omega_{k i}^{\top}(t, \beta) \Theta_{k i} \omega_{k i}(t, \beta) \mathrm{d} \beta\right]
\end{aligned}
$$

where $\omega_{k i}^{\top}(t, \beta)=\left(y_{k i}^{\top}(t), y_{k i}^{\top}(t-d(t)), \dot{y}_{k i}^{\top}(\beta)\right)^{\top}$, and

$$
\hat{\Phi}_{k i}=\left(\begin{array}{cc}
\phi_{11}^{k i}+\tau A^{\top}(t) Z_{k i} A(t) & \phi_{12}^{k i}+\tau \lambda_{k i} A^{\top}(t) Z_{k i} \Gamma_{k} \\
* & \phi_{22}^{k i}+\tau \lambda_{k i}^{2} \Gamma_{k}^{\top} Z_{k i} \Gamma_{k}
\end{array}\right) .
$$

By using Lemma 1 and (13) we get $\hat{\Phi}_{k i}<0$. Thus, it follows from (14) and (24) that

$$
\dot{V}(t)+\alpha V(t) \leq 0 .
$$

Integrating the above inequality on the interval $\left[t_{r}, t\right], \forall t \in$ $\left[t_{r}, t_{r+1}\right)$ gives

$$
V(t)=V_{\sigma(t)}(t) \leq \mathrm{e}^{-\alpha\left(t-t_{r}\right)} V_{\sigma\left(t_{r}\right)}\left(t_{r}\right) .
$$

When at the switching instant, i.e., $t=t_{r}$, from (16), (17) and (18), we have

$$
V_{\sigma\left(t_{r}^{+}\right)}\left(t_{r}^{+}\right) \leq \mu V_{\sigma\left(t_{r}^{-}\right)}\left(t_{r}^{-}\right), \quad r=1,2, \ldots
$$

Therefore, it follows from (25), (26) and the relation $q=N_{\sigma}\left(t_{0}, t\right) \leq N_{0}+\left(t-t_{0}\right) / T_{a}$ that

$$
\begin{aligned}
V(t) & \leq \mathrm{e}^{-\alpha\left(t-t_{q}\right)} \mu V_{\sigma\left(t_{q}^{-}\right)}\left(t_{q}^{-}\right) \\
& \leq \cdots \leq \mathrm{e}^{-\alpha\left(t-t_{0}\right)} \mu^{q} V_{\sigma\left(t_{0}\right)}\left(t_{0}\right) \\
& \leq \mu^{N_{0}} \mathrm{e}^{-\left(\alpha-\frac{\ln \mu}{T_{a}}\right)\left(t-t_{0}\right)} V_{\sigma\left(t_{0}\right)}\left(t_{0}\right) .
\end{aligned}
$$

According to (17) and (18), we have

$$
V(t) \geq a\|e(t)\|^{2}, \quad V\left(t_{0}\right) \leq b\left\|e\left(t_{0}\right)\right\|_{c l}^{2}
$$

where $\|\cdot\|$ is the Euclidean norm, $\|e(t)\|_{c l}=$ $\sup _{-\tau<\theta<0}\{\|e(t+\theta)\|,\|\dot{e}(t+\theta)\|\}, \quad a=$ $\min _{\forall k \in \mathcal{M}} \lambda_{\min }\left(\tilde{P}_{k}\right), \quad b=\max _{\forall k \in \mathcal{M}} \lambda_{\max }\left(\tilde{P}_{k}\right)+$ $\tau \max _{\forall k \in \mathcal{M}} \lambda_{\max }\left(\tilde{Q}_{k}\right)+\left(\tau^{2} / 2\right) \max _{\forall k \in \mathcal{M}} \lambda_{\max }\left(\tilde{Z}_{k}\right)$,

$\lambda_{\min }\left(\tilde{P}_{k}\right)$ and $\lambda_{\max }\left(\tilde{P}_{k}\right)$ are the minimum and maximum eigenvalue of $\tilde{P}_{k}$, respectively.

Combining (27) and (28), leads to

$$
\|e(t)\|^{2} \leq \frac{1}{a} V(t) \leq \frac{b}{a} \mu^{N_{0}} \mathrm{e}^{-\left(\alpha-\frac{\ln \mu}{T_{a}}\right)\left(t-t_{0}\right)}\left\|e\left(t_{0}\right)\right\|_{c l}^{2} .
$$

Therefore, we get that the synchronization manifold $\mathbb{S}$ of delayed dynamical network (1) with switching topology is locally exponentially stable. The proof is thus completed.

When $Q_{k i}=0$ in (13), the constraint on the derivative of $d(t)$ is removed, and the conditions obtained in Theorem 1 degenerate into delay-derivative-free ones. In this case, the derivative of $d(t)$ can be unknown or arbitrary. This is addressed in Corollary 1.

Corollary 1: Suppose Assumption 2 holds, and if (13) with $Q_{k i}=0$ and (14) hold, then the synchronous manifold $\mathbb{S}$ of the delayed network (1) is locally exponentially stable for any switching signal with average dwell time satisfying (15).

Proof: Choose the Lyapunov functional candidate $V(t)=V_{\sigma(t)}(t)=V_{1 \sigma(t)}+V_{2 \sigma(t)}$ as described in (17) and (18). The proof procedure is similar to that of Theorem 1, and it is omitted.

Remark 2: It is worth mentioning that even though synchronization is reached for each individual topology, synchronization may fail for switching topology (see Example 1 for an instance that the relatively fast switching between two exponentially synchronized subnetwork will destroy the synchronizability of the overall switched network). One way to have synchronization with the switching topology is to constrain the switching signal $\sigma(t)$ by the average dwell time (15). As a special case, if $\mu=1$ in Theorem 1, (16) implies that $V=V_{k}, \forall k \in$ $\mathcal{M}$, is a common Lyapunov functional for the switched system (8). Therefore, the switched system is exponentially stable for arbitrary switching which means that the synchronization manifold of the network (1) is locally exponentially stable for arbitrary switching.

Remark 3: In this paper, we do not require a common transform matrix $U$ for all the different outer coupling matrices $C_{k}$ which is needed in [30]. Obviously, this requirement is far too strong and unrealistic. We also do not require common matrices $P_{k}, Q_{k}$ and $Z_{k}$ for the index $i$ in inequality (13) and (14), this reduces the conservativeness of obtained results and meanwhile also makes the average dwell time proposed in Theorem 1 depend on the transform matrices $U_{k}$. Furthermore, if there exit common matrices $P_{k} \in \mathbb{R}^{n \times n}, Q_{k} \in \mathbb{R}^{n \times n}$ and $Z_{k} \in \mathbb{R}^{n \times n}$ in (13) and (14) for the index $i$, then we can select the Lyapunov functional as

$$
\begin{aligned}
V_{\sigma(t)}(t)= & e^{\top}(t)\left(I_{N} \otimes P_{\sigma(t)}\right) e(t) \\
& +\int_{t-d(t)}^{t} \mathrm{e}^{\alpha(\beta-t)} e^{\top}(\beta)\left(I_{N} \otimes Q_{\sigma(t)}\right) e(\beta) \mathrm{d} \beta \\
& +\int_{-\tau}^{0} \int_{t+\theta}^{t} \mathrm{e}^{\alpha(\beta-t)} \dot{e}^{\top}(\beta)\left(I_{N} \otimes Z_{\sigma(t)}\right) \dot{e}(\beta) \mathrm{d} \beta \mathrm{d} \theta .
\end{aligned}
$$

In this case, the differences of the values of Lyapunov functional $V_{\sigma(t)}$ at the switching instants are independent of the different transformation matrices $U_{k}$, which finally lead the average dwell time to be independent of $U_{k}$.

Remark 4: In the literature, many papers assumed that the investigated network has the symmetric and positively weighted outer coupling matrix $\left(c_{i j}=c_{j i} \geq 0\right)$ as we did in Section II-see, for example, [10], [13], [20], and [35]. However, the positively weighted condition on the network topology can be removed here. We can only require symmetry of the outer coupling matrix, i.e., if there is a connection between two nodes $i$ and $j$, then $c_{i j}=c_{j i} \neq 0$, otherwise $c_{i j}=c_{j i}=0$. This means that positive and negative connections can coexist in a network. The existence of the negative connections may lead to some positive eigenvalues of the outer coupling matrix. But, the zero row sum condition (2) guarantees that $C_{k}$ has at least one zero eigenvalue with associated eigenvector $u_{k 1}=1 / \sqrt{N}(1,1, \ldots, 1)^{\top}$. Rearranging the remaining eigenvalues and the corresponding eigenvectors will still make (10), (11), and (12) hold. So if all conditions proposed in Theorem 1 are satisfied, then synchronization of the switched network under the average dwell time switching law is guaranteed. On 
the other hand, our results can also apply to some special kind of nonsymmetric networks. If all candidate outer coupling matrices $C_{k}, \forall k \in \mathcal{M}$ are similar to diagonal matrices having real eigenvalues and real eigenvectors, then by replacing the unitary matrix $U_{k}$ by a nonsingular matrix $\Phi_{k}$, a similar result as Theorem 1 can be obtained for this kind of networks. However, how to extend the result to the general nonsymmetric network deserves more attention in future work.

Remark 5: Conditions (13) and (14) guarantee that all the subnetworks in (3) are exponentially synchronized at least with a given exponential rate $\alpha$, i.e., $\|e(t)\|^{2} \leq a_{0} \mathrm{e}^{-\alpha\left(t-t_{0}\right)}\left\|e\left(t_{0}\right)\right\|_{c l}^{2}$ where $a_{0}$ is a constant associated with $\tilde{P}_{k}, \tilde{Q}_{k}, \tilde{Z}_{k}$ and $\tau$. But this estimation is not the best one. For each error dynamical system (9), we can solve LMIs (13) and (14) repeatedly with giving different values of $\alpha$ to get a better estimation of the convergence rate $\alpha_{k}$. The conservativeness of this estimation mainly comes from the inequalities scaling in the proof procedure of Theorem 1. For example, we use $e^{-\alpha \tau}$ to replace $e^{\alpha(\beta-t)},(t-\tau \leq$ $\beta \leq t)$ in inequality (20), and use $(1-d) e^{-\alpha \tau}$ to replace $(1-\dot{d}(t)) e^{-\alpha d(t)}$ in (21). So when $\tau$ and $d$ are small enough, $\alpha_{k}$ is close to its truth value $\alpha_{k}^{*}$; otherwise, the difference between $\alpha_{k}$ and $\alpha_{k}^{*}$ will increase as $\tau$ and $d$ becoming large. For the switched network, this conservativeness will be enlarged, because we can not sufficiently use the convergence rate $\alpha_{k}$ of each subnetwork that we can get, but use $\alpha=\min _{k \in \mathcal{M}}\left\{\alpha_{k}\right\}$ instead to get the positive definite matrices $P_{k i}, Q_{k i}, Z_{k i}$ by solving LMIs (13) and (14), which are used to compute $\mu$ and finally lead to the bound of the average dwell time $T_{a}^{*}$.

Remark 6: For the bound of the average dwell time $T_{a}^{*}$, from the formula (15), we see that the larger $\alpha$, the smaller $T_{a}^{*}$. Since $T_{a}^{*}$ depends on $\alpha$, the conservativeness for estimating $\alpha$ will transmit to the estimation of $T_{a}^{*}$. As we mentioned in Remark 5 , we can not sufficiently use each subnetwork's own decay rate $\alpha_{k}$, this will definitely introduce some conservativeness into the bound estimating for the average dwell time $T_{a}^{*}$. However, what we are trying to do is to give a safety bound of $T_{a}^{*}$ which can give a theoretical guide to the practice.

\section{B. Some Subnetworks Are Self-Synchronizing}

In the previous subsection, we assumed that all subnetworks are self-synchronizing. In this subsection, we consider a more general case, that is, the switched network (1) consists of both self-synchronizing and nonsynchronizing subnetworks. We investigate how to tolerate the existence of nonsynchronizing subnetworks without destroying synchronizability of the overall network. Without loss of generality, we suppose that $k \in \mathcal{M}^{-}$ denote the index of the self-synchronizing subnetworks, while $k \in \mathcal{M}^{+}$denote the index of the nonsynchronizing subnetworks. Thus, $\mathcal{M}^{-} \cup \mathcal{M}^{+}=\mathcal{M}$.

In order to eliminate the impact on the overall network caused by the nonsynchronizing subnetworks, the average dwell time as a constraint on the switching signal is not sufficient. We have to find further condition on the switching signal to guarantee the synchronizability of the overall network. Let $T^{-}(t)$ (resp., $\left.T^{+}(t)\right)$ denote the total activation time of the self-synchronizing subnetworks (resp. the nonsynchronizing subnetworks). Let $\alpha^{-}$ and $\alpha^{+}$be positive numbers to be selected later. For any given $\alpha \in\left(0, \alpha^{-}\right)$, we choose an arbitrary $\alpha^{*} \in\left(\alpha, \alpha^{-}\right)$and propose the switching law satisfying the following condition [41]:

$$
\inf _{t \geq t_{0}} \frac{T^{-}(t)}{T^{+}(t)} \geq \frac{\alpha^{+}+\alpha^{*}}{\alpha^{-}-\alpha^{*}}
$$

Theorem 2: For given constants $\alpha^{-}>0$ and $\alpha^{+}>0$, suppose Assumption 1 holds and there exist matrices $P_{k i}>0$, $Q_{k i}>0, Z_{k i}>0, Y_{k i}, T_{k i}$ and

$$
X_{k i}=\left(\begin{array}{cc}
X_{11}^{k i} & X_{12}^{k i} \\
* & X_{22}^{k i}
\end{array}\right) \geq 0
$$

with appropriate dimensions such that

$$
\begin{aligned}
& \Phi_{k i}^{-}=\left(\begin{array}{ccc}
\phi_{11}^{k i-} & \phi_{12}^{k i} & \tau A^{\top}(t) Z_{k i} \\
* & \phi_{22}^{k i-} & \tau \lambda_{k i} \Gamma_{k}^{\top} Z_{k i} \\
* & * & -\tau Z_{k i}
\end{array}\right)<0, \\
& i \in \mathcal{N}_{1}, \quad k \in \mathcal{M}^{-} \text {, } \\
& \Phi_{k i}^{+}=\left(\begin{array}{ccc}
\phi_{11}^{k i+} & \phi_{12}^{k i} & \tau A^{\top}(t) Z_{k i} \\
* & \phi_{22}^{k i+} & \tau \lambda_{k i} \Gamma_{k}^{\top} Z_{k i} \\
* & * & -\tau Z_{k i}
\end{array}\right)<0, \\
& i \in \mathcal{N}_{1}, \quad k \in \mathcal{M}^{+} \text {, } \\
& \begin{aligned}
\Theta_{k i}= & \left(\begin{array}{ccc}
X_{11}^{k i} & X_{12}^{k i} & Y_{k i} \\
* & X_{22}^{k i} & T_{k i} \\
* & * & \mathrm{e}^{-\tilde{\alpha} \tau} Z_{k i}
\end{array}\right) \geq 0, \\
& i \in \mathcal{N}_{1}, \quad k \in \mathcal{M}
\end{aligned}
\end{aligned}
$$

where

$$
\begin{aligned}
\phi_{11}^{k i-}= & P_{k i} A(t)+A^{\top}(t) P_{k i}+Y_{k i}^{\top}+Y_{k i}+Q_{k i}+\tau X_{11}^{k i} \\
& +\alpha^{-} P_{k i}, \\
\phi_{22}^{k i-}= & -T_{k i}-T_{k i}^{\top}-(1-d) \mathrm{e}^{-\alpha^{-} \tau} Q_{k i}+\tau X_{22}^{k i}, \\
\phi_{11}^{k i+}= & P_{k i} A(t)+A^{\top}(t) P_{k i}+Y_{k i}^{\top}+Y_{k i}+Q_{k i}+\tau X_{11}^{k i} \\
& -\alpha^{+} P_{k i}, \\
\phi_{22}^{k i+}= & -T_{k i}-T_{k i}^{\top}-(1-d) Q_{k i}+\tau X_{22}^{k i}
\end{aligned}
$$

and $\tilde{\alpha}=\alpha^{-}$for $k \in \mathcal{M}^{-}$, otherwise $\tilde{\alpha}=0$. Then the synchronous manifold $\mathbb{S}$ of the delayed network (1) is locally exponentially stable for any switching signal satisfying the condition (29) and the average dwell time

$$
T_{a} \geq T_{a}^{*}=\frac{\ln \mu}{\alpha^{*}-\alpha}
$$

where $\mu$ and $\phi_{12}^{k i}$ are defined the same as in Theorem 1 .

Proof: For $k \in \mathcal{M}^{-}$, select the piecewise Lyapunov functional candidate (17) and (18) by replacing $\alpha$ by $\alpha^{-}$. While for $k \in \mathcal{M}^{+}$, select the following piecewise Lyapunov functional candidate:

$$
\begin{aligned}
V(t)= & V_{\sigma(t)}(t)=e^{\top}(t) \tilde{P}_{\sigma(t)} e(t) \\
& +\int_{t-d(t)}^{t} \mathrm{e}^{\alpha^{+}(t-\beta)} e^{\top}(\beta) \tilde{Q}_{\sigma(t)} e(\beta) \mathrm{d} \beta \\
& +\int_{-\tau}^{0} \int_{t+\theta}^{t} \mathrm{e}^{\alpha^{+}(t-\beta)} \dot{e}^{\top}(\beta) \tilde{Z}_{\sigma(t)} \dot{e}(\beta) \mathrm{d} \beta \mathrm{d} \theta .
\end{aligned}
$$

Let $\sigma(t)=k$ for $t \in\left[t_{r}, t_{r+1}\right)$. Similar to (19)-(25), when $t \in\left[t_{r}, t_{r+1}\right)$, we have

$$
V(t)=V_{\sigma(t)}(t)=V_{k}(t) \leq \begin{cases}\mathrm{e}^{-\alpha^{-}\left(t-t_{r}\right)} V_{k}\left(t_{r}\right) & k \in \mathcal{M}^{-}, \\ \mathrm{e}^{\alpha^{+}\left(t-t_{r}\right)} V_{k}\left(t_{r}\right) & k \in \mathcal{M}^{+}\end{cases}
$$


With the same procedure as in Theorem 1, we have the inequality (26) when $t=t_{r}$. Using (26), (35) and $N_{\sigma}\left(t_{0}, t\right) \leq$ $N_{0}+\left(t-t_{0}\right) / T_{a}$ lead to

$$
\begin{aligned}
V(t) & \leq \mu^{N_{\sigma}\left(t_{0}, t\right)} \mathrm{e}^{\alpha^{+} T^{+}(t)-\alpha^{-} T^{-}(t)} V_{\sigma\left(t_{0}\right)}\left(t_{0}\right) \\
& \leq \mu^{N_{0}} \mathrm{e}^{\alpha^{+} T^{+}(t)-\alpha^{-} T^{-}(t)+\frac{t-t_{0}}{T_{a}} \ln \mu} V_{\sigma\left(t_{0}\right)}\left(t_{0}\right) .
\end{aligned}
$$

Combining (29), (33) and (36), we get

$$
\begin{aligned}
& V(t) \leq \mu^{N_{0}} \mathrm{e}^{-\alpha^{*}\left(T^{+}(t)+T^{-}(t)\right)+\frac{t-t_{0}}{T_{a}} \ln \mu} V_{\sigma\left(t_{0}\right)}\left(t_{0}\right) \\
& =\mu^{N_{0}} \mathrm{e}^{-\left(\alpha^{*}-\frac{\ln \mu}{T_{a}}\right)\left(t-t_{0}\right)} V_{\sigma\left(t_{0}\right)}\left(t_{0}\right) \\
& \leq \mu^{N_{0}} \mathrm{e}^{-\alpha\left(t-t_{0}\right)} V_{\sigma\left(t_{0}\right)}\left(t_{0}\right) \text {. }
\end{aligned}
$$

Therefore, we have

$$
\|e(t)\|^{2} \leq \frac{b}{a} \mu^{N_{0}} \mathrm{e}^{-\alpha\left(t-t_{0}\right)}\left\|e\left(t_{0}\right)\right\|_{c l}^{2} .
$$

where $a, b$ and $\|e(t)\|_{c l}$ are defined the same as in Theorem 1. This implies that the switched system (8) is exponentially stable for any switching signal satisfying (29) and average dwell time (33). Thus, we get that the synchronous solution of dynamical network (1) with switching topology is locally exponentially stable. This completes the proof.

Similar to Corollary 1, we have the following criterion for the delay-derivative-free conditions.

Corollary 2: Suppose Assumption 2 holds, and if (30) and (31) both with $Q_{k i}=0$ and (32) hold, then the synchronous manifold $\mathbb{S}$ of the delayed network (1) is locally exponentially stable for any switching signal satisfying the condition (29) and the average dwell time (33).

Proof: The proof is similar to that of Theorem 2.

Remark 7: It is well known that a suitable average dwell time can overcome the influence on the switched system caused by switching between different stable subsystems [38]. So the average dwell time is sufficient to guarantee the stability of the synchronous states of the overall network when all subnetworks in (3) are self-synchronizing. However, this is not the case when there are some nonsynchronizing subnetworks in (3). The existence of nonsynchronizing subnetworks may destroy the synchronizability of the overall network. To overcome it, we have to further specify the switching signal in addition to average dwell time, i.e., constrain the ratio of the total activation time of self-synchronizing and nonsynchronizing subnetworks by inequality (29). The essence of this method is to activate the self-synchronizing subnetworks a relatively long period to counteract the increment of the value of Lyapunov functional along the overall network caused by nonsynchronizing subnetworks.

Remark 8: To reduce the conservativeness and get a better estimation of $\alpha^{+}$, we select different Lyapunov functionals for the nonsynchronizing subnetworks from the ones for the self-synchronizing subnetworks by using $e^{\alpha^{+}(t-\beta)}$ instead of $e^{\alpha^{-}(\beta-t)}$ in (18). The main conservativeness of the ratio bound (29) is also from the inequalities scaling when deducing the LMIs (30), (31) and (32). Here, we should note that $\alpha^{*} \in\left(\alpha, \alpha^{-}\right)$is a designable parameter, so we can adjust the ratio (29) to a desirable one to some extent by choosing distinct values of $\alpha^{*}$. But on the other hand, this parameter is also associated with the computation of $T_{a}^{*}$, which is to say that one has to balance the ratio bound (29) and the average dwell time bound $T_{a}^{*}$ by tuning $\alpha^{*}$ in its admissible set.

Remark 9: As we mentioned in the introduction, fast switching is not allowed in some real-world networks, and may shorten the actuator's working period. In this case, the average dwell time switching algorithm is preferable. But on the other hand, average dwell time method can only apply to the case where there is at least one self-synchronizing subnetwork. This will prevent its application to the case where all subnetworks are nonsynchronizing. When this situation happens, the average dwell time method will lose its power without auxiliary equipment such as adding controllers to synchronize one of the subnetworks. For the latter case, if fast switching is allowable, then the synchronization of switched network may still be achieved by sufficient fast switching between the candidate subnetworks [25].

\section{GLOBAL EXPONENTIAL SyNCHRONIZATION}

We consider the global synchronization problem in this section. We only focus on a particular form of the delayed network model

$$
\begin{aligned}
\dot{x}_{i}(t)=A x_{i}(t) & +f\left(x_{i}(t)\right) \\
& +\sum_{j=1}^{N} c_{i j}^{\sigma(t)} \Gamma_{\sigma(t)} x_{j}(t-d(t)), \quad i \in \mathcal{N} .
\end{aligned}
$$

Obviously, the switched network (38) is composed by a switching signal $\sigma(t)$ and the following family of subnetworks:

$$
\begin{array}{r}
\dot{x}_{i}(t)=A x_{i}(t)+f\left(x_{i}(t)\right)+\sum_{j=1}^{N} c_{i j}^{k} \Gamma_{k} x_{j}(t-d(t)), \\
i \in \mathcal{N}, \quad k \in \mathcal{M}
\end{array}
$$

where $A$ is a constant matrix, $C_{k}, \Gamma_{k}$ and $d(t)$ are defined the same as in Section II; $f(\cdot): \mathbb{R}^{n} \rightarrow \mathbb{R}^{n}$ is a continuous nonlinear function satisfying

$$
\left\|f\left(x_{i}(t)\right)-f(s(t))\right\| \leq \gamma\left\|x_{i}(t)-s(t)\right\|, \quad i \in \mathcal{N}
$$

where $\gamma>0$ is a positive constant, $s(t)=(1 / N) \sum_{i=1}^{N} x_{i}(t)$ Then the error systems are

$$
\begin{aligned}
\dot{e}_{i}(t)=A e_{i}(t) & +f\left(x_{i}(t)\right)-f(s(t)) \\
& +\sum_{j=1}^{N} c_{i j}^{\sigma(t)} \Gamma_{\sigma(t)} e_{j}(t-d(t))+J, \quad i \in \mathcal{N}
\end{aligned}
$$

where $J=f(s(t))-(1 / N) \sum_{p=1}^{N} f\left(x_{p}(t)\right)$.

Rewrite (41) as

$$
\dot{e}(t)=\bar{A} e(t)+\bar{C}_{\sigma(t)} e(t-d(t))+F+\bar{J}
$$

where $\bar{A}=I_{N} \otimes A, \bar{C}_{\sigma(t)}=C_{\sigma(t)} \otimes \Gamma_{\sigma(t)} F=F(e, s, t)=$ $\left(\left(f\left(x_{1}\right)-f(s)\right)^{\top},\left(f\left(x_{2}\right)-f(s)\right)^{\top}, \ldots,\left(f\left(x_{N}\right)-f(s)\right)^{\top}\right)^{\top} \in$ $\mathbb{R}^{n N}$, and $\bar{J}=\left(J^{\top}, J^{\top}, \ldots, J^{\top}\right)^{\top} \in \mathbb{R}^{n N}$.

In the following, as in Section III, we also discuss the problem in two cases, and give two global exponential synchronization criteria for the switched network (38).

\section{A. All Subnetworks Are Self-Synchronizing}

When all subnetworks in (39) are exponentially synchronized, we have the following theorem.

Theorem 3: Consider the switched network (38). For a given constant $\alpha>0$, suppose Assumption 1 holds, and if there exist a constant $\varepsilon>0$ and matrices $P_{k i}>0, Q_{k i}>0, Z_{k i}>0, Y_{k i}$, $T_{k i}$, and

$$
X_{k i}=\left(\begin{array}{cc}
X_{11}^{k i} & X_{12}^{k i} \\
* & X_{22}^{k i}
\end{array}\right) \geq 0
$$


with appropriate dimensions such that

$$
\begin{aligned}
\Phi_{k i}= & \left(\begin{array}{cccc}
\phi_{11}^{k i} & \phi_{12}^{k i} & P_{k i} & \tau A^{\top} Z_{k i} \\
* & \phi_{22}^{k i} & 0 & \tau \lambda_{k i} \Gamma_{k}^{\top} Z_{k i} \\
* & * & -\varepsilon I_{n} & \tau Z_{k i} \\
* & * & * & -\tau Z_{k i}
\end{array}\right)<0, \\
& i \in \mathcal{N}_{1}, \quad k \in \mathcal{M}, \\
\Theta_{k i}= & \left(\begin{array}{ccc}
X_{11}^{k i} & X_{12}^{k i} & Y_{k i} \\
* & X_{22}^{k i} & T_{k i} \\
* & * & \mathrm{e}^{-\alpha \tau} Z_{k i}
\end{array}\right) \geq 0 \\
& i \in \mathcal{N}_{1}, \quad k \in \mathcal{M}
\end{aligned}
$$

where

$$
\begin{aligned}
\phi_{11}^{k i}= & P_{k i} A+A^{\top} P_{k i}+Y_{k i}^{\top}+Y_{k i}+Q_{k i}+\tau X_{11}^{k i}+\varepsilon \gamma^{2} I_{n} \\
& +\alpha P_{k i}, \\
\phi_{12}^{k i}= & \lambda_{k i} P_{k i} \Gamma_{k}-Y_{k i}+T_{k i}^{\top}+\tau X_{12}^{k i}, \\
\phi_{22}^{k i}= & -T_{k i}-T_{k i}^{\top}-(1-d) \mathrm{e}^{-\alpha \tau} Q_{k i}+\tau X_{22}^{k i}
\end{aligned}
$$

then the synchronization manifold $\mathbb{S}$ is globally exponentially stable for any switching signal with average dwell time satisfying

$$
T_{a}>T_{a}^{*}=\frac{\ln \mu}{\alpha}
$$

where $\mu \geq 1$ satisfies

$$
\tilde{P}_{k} \leq \mu \tilde{P}_{l}, \quad \tilde{Q}_{k} \leq \mu \tilde{Q}_{l}, \quad \tilde{Z}_{k} \leq \mu \tilde{Z}_{l}, \quad \forall k, l \in \mathcal{M}
$$

and $\tilde{P}_{k}, \tilde{Q}_{k}, \tilde{Z}_{k}$ are defined the same as in Theorem 1, but with $Z_{k 1}=\mathbf{0} \in \mathbb{R}^{n \times n}$.

Proof: Select the piecewise Lyapunov functional candidate (17), and on each interval $\left[t_{r}, t_{r+1}\right)$, let $\sigma(t)=k$. Obviously, $\forall t \in\left[t_{r}, t_{r+1}\right)$ we have

$$
\begin{aligned}
\dot{V}_{1 k}(t)=y_{k}^{\top}(t) & {\left[\bar{A}^{\top} \bar{P}_{k}+\bar{P}_{k} \bar{A}\right] y_{k}(t) } \\
+ & +2 y_{k}^{\top}(t) \bar{P}_{k}\left[\bar{\Lambda}_{k} y_{k}(t-d(t))+G_{k}+\tilde{J}\right]
\end{aligned}
$$

where $G_{k}=G_{k}(e, s, t)=\left(U_{k}^{\top} \otimes I_{n}\right) F(e, s, t)=$ $\left(G_{k 1}^{\top}, G_{k 2}^{\top}, \ldots, G_{k N}^{\top}\right)^{\top}$ with $G_{k i}=\left(u_{k i}^{\top} \otimes I_{n}\right) F, \tilde{J}=$ $\left(U_{k}^{\dagger} \otimes I_{n}\right) \bar{J}$.

Let $W=y_{k}^{\top}(t) \bar{P}_{k} \tilde{J}$. Due to the fact that $U_{k}$ is a unitary matrix with $u_{k 1}=\left(u_{11}^{k}, u_{21}^{k}, \ldots, u_{N 1}^{k}\right)^{\top}=$ $(1 / \sqrt{N})(1,1, \ldots, 1)^{\top}$ for all $k \in \mathcal{M}$, we have

$$
\begin{cases}\sum_{j=1}^{N} u_{j i}^{k}=\sqrt{N}, & i=1, k \in \mathcal{M} \\ \sum_{j=1}^{N} u_{j i}^{k}=0, & i \in \mathcal{N}_{1}, k \in \mathcal{M} .\end{cases}
$$

With $y_{k 1} \equiv \mathbf{0} \in \mathbb{R}^{n}$, then one gets

$$
\begin{aligned}
W= & \left(y_{k 1}^{\top}, y_{k 2}^{\top}, \ldots, y_{k N}^{\top}\right) \bar{P}_{k}\left(U_{k}^{\top} \otimes I_{n}\right) \bar{J} \\
= & \left(\mathbf{0}^{\top}, y_{k 2}^{\top}, \ldots, y_{k N}^{\top}\right) \bar{P}_{k} \\
& \times\left(\sum_{j=1}^{N} u_{j 1}^{k} J^{\top}, \sum_{j=1}^{N} u_{j 2}^{k} J^{\top}, \ldots, \sum_{j=1}^{N} u_{j N}^{k} J^{\top}\right)^{\top} \\
= & 0 .
\end{aligned}
$$

So we have

$$
\begin{aligned}
\dot{V}_{1 k}(t)= & y_{k}^{\top}(t)\left[\bar{A}^{\top} \bar{P}_{k}+\bar{P}_{k} \bar{A}\right] y_{k}(t) \\
& +2 y_{k}^{\top}(t) \bar{P}_{k}\left[\bar{\Lambda}_{k} y_{k}(t-d(t))+G_{k}\right] .
\end{aligned}
$$

$$
\begin{aligned}
\dot{V}_{2 k} \leq & -\alpha V_{2 k}+\tau \dot{y}_{k}^{\top}(t) \bar{Z}_{k} \dot{y}_{k}(t) \\
& -\int_{t-d(t)}^{t} \mathrm{e}^{-\alpha \tau} \dot{y}_{k}^{\top}(\beta) \bar{Z}_{k} \dot{y}_{k}(\beta) \mathrm{d} \beta \\
= & -\alpha V_{2 k}+\tau\left[\bar{A} y_{k}(t)+\bar{\Lambda}_{k} y_{k}(t-d(t))+G_{k}\right]^{\top} \\
& \times \bar{Z}_{k}\left[\bar{A} y_{k}(t)+\bar{\Lambda}_{k} y_{k}(t-d(t))+G_{k}\right] \\
& -\int_{t-d(t)}^{t} \mathrm{e}^{-\alpha \tau} \dot{y}_{k}^{\top}(\beta) \bar{Z}_{k} \dot{y}_{k}(\beta) \mathrm{d} \beta,
\end{aligned}
$$

where we use the properties that $y_{k}^{\top}(t) \bar{A}^{\top} \bar{Z}_{k} \tilde{J}=y_{k}^{\top}(t-$ $d(t)) \bar{\Lambda}_{k}^{\top} \bar{Z}_{k} \tilde{J}=G_{k}^{\top} \bar{Z}_{k} \tilde{J}=\tilde{J}^{\top} \bar{Z}_{k} \tilde{J}^{k}=0$, which are followed by $y_{k 1} \equiv \mathbf{0}$ and $Z_{k 1}=\mathbf{0}$ directly. Moreover, we have

$$
\begin{aligned}
\dot{V}_{3 k} \leq-\alpha V_{3 k}+y_{k}^{\top}(t) & \bar{Q}_{k} y_{k}(t)-(1-d) \mathrm{e}^{-\alpha \tau} \\
& \times y_{k}^{\top}(t-d(t)) \bar{Q}_{k} y_{k}(t-d(t)) .
\end{aligned}
$$

From (40), we have $G_{k}^{\top} G_{k}=F^{\top} F \leq \gamma^{2} e^{\top}(t) e(t)=$ $\gamma^{2} y_{k}^{\top}(t) y_{k}(t)$. By S-Procedure [43], we get

$$
\dot{V}_{k} \leq \dot{V}_{1 k}+\dot{V}_{2 k}+\dot{V}_{3 k}-\varepsilon\left[G_{k}^{\top} G_{k}-\gamma^{2} y_{k}^{\top}(t) y_{k}(t)\right] .
$$

With the same procedure as in Theorem 1 and $y_{k 1} \equiv \mathbf{0}, Z_{k 1}=$ $\mathbf{0}$, we have

$$
\begin{aligned}
\dot{V}(t)+\alpha V(t) \leq \sum_{i=2}^{N} & {\left[\varpi_{k i}^{\top}(t) \hat{\Phi}_{k i} \varpi_{k i}(t)\right.} \\
& \left.-\int_{t-d(t)}^{t} \omega_{k i}^{\top}(t, \beta) \Theta_{k i} \omega_{k i}(t, \beta) \mathrm{d} \beta\right]
\end{aligned}
$$

where $\varpi_{k i}(t)=\left(y_{k i}^{\top}(t), y_{k i}^{\top}(t-d(t)), G_{k i}^{\top}\right)^{\top}, \omega_{k i}(t, \beta)=$ $\left(y_{k i}^{\top}(t), y_{k i}^{\top}(t-d(t)), \dot{y}_{k i}^{\top}(\beta)\right)^{\top}$, and

$$
\hat{\Phi}_{k i}=\left(\begin{array}{ccc}
\phi_{11}^{k i}+\tau A^{\top} Z_{k i} A & \phi_{12}^{k i}+\tau \lambda_{k i} A^{\top} Z_{k i} \Gamma_{k} & P+\tau A^{\top} Z_{k i} \\
* & \phi_{22}^{k i}+\tau \lambda_{k i}^{2} \Gamma_{k}^{\top} Z_{k i} \Gamma_{k} & \tau \lambda_{k i} \Gamma_{k}^{\top} Z_{k i} \\
* & * & \tau Z_{k i}-\varepsilon I_{n}
\end{array}\right) .
$$

By Lemma 1 and (43), we have $\hat{\Phi}_{k i}<0$ and

$$
\dot{V}(t)+\alpha V(t)<0 \text {. }
$$

Thus, we have

$$
\|e(t)\|^{2} \leq \frac{b}{a} \mu^{N_{0}} \mathrm{e}^{-\left(\alpha-\frac{\ln \mu}{T_{a}}\right)\left(t-t_{0}\right)}\left\|e\left(t_{0}\right)\right\|_{c l}^{2}
$$

where $a, b$, and $\|e(t)\|_{c l}$ are defined as in Theorem 1. Therefore, we get that the synchronization manifold $\mathbb{S}$ of dynamical network (38) with switching topology is globally exponentially stable. The proof is completed.

Remark 10: Generally, even though there does exit a $\bar{\mu} \geq 1$ satisfying $\bar{Z}_{k} \leq \bar{\mu} \bar{Z}_{l}$ for positive semidefinite matrices $\bar{Z}_{k}$ and $\bar{Z}_{l}$ defined as in Theorem 3, it is hard to say whether there exists a $\mu \geq 1$ such that $\tilde{Z}_{k} \leq \mu \tilde{Z}_{l}$ for arbitrary different unitary matrices $U_{k}$ and $U_{l}$, because the unitary transformations $U_{k} \otimes I_{n}$ and $U_{l} \otimes I_{n}$ may change the zero eigenvalues of $\tilde{Z}_{k}$ and $\tilde{Z}_{l}$ into different positions. However, in our cases, we do not have such a problem. Due to the special property (2) of the outer coupling matrix $C_{k}$, we have $u_{1}=u_{k 1}=(1 / \sqrt{N})(1,1, \ldots, 1)^{\top}$. By 
simple calculation, we can get that there exists a unitary matrix $\Psi=\left(\psi_{1}, \psi_{2}, \ldots, \psi_{N}\right) \in \mathbb{R}^{N \times N}$ with $\psi_{1}=u_{1}$ such that

$$
\left(\Psi^{\top} \otimes I_{n}\right) \tilde{Z}_{k}\left(\Psi \otimes I_{n}\right)=\left(\begin{array}{cc}
\mathbf{0}_{n \times n} & \mathbf{0}_{n \times(N-1) n} \\
* & \hat{Z}_{k}
\end{array}\right)
$$

with $\hat{Z}_{k}>0, \forall k \in \mathcal{M}$. Then there exists a $\mu \geq 1$ such that $\hat{Z}_{k} \leq \mu \hat{Z}_{l}$ which is equivalent to $\tilde{Z}_{k} \leq \mu \tilde{Z}_{l}$.

\section{B. Some Subnetworks Are Self-Synchronizing}

Here, we consider global exponential synchronization of the network (38) which is composed of both self-synchronizing and nonsynchronizing subnetworks in (39). The criterion is stated as follows.

Theorem 4: Consider the switched network (38). For given constants $\alpha^{-}>0$ and $\alpha^{+}>0$, suppose Assumption 1 holds, and if there exist a constant $\varepsilon>0$ and matrices $P_{k i}>0, Q_{k i}>$ $0, Z_{k i}>0, Y_{k i}, T_{k i}$ and

$$
X_{k i}=\left(\begin{array}{cc}
X_{11}^{k i} & X_{12}^{k i} \\
* & X_{22}^{k i}
\end{array}\right) \geq 0
$$

with appropriate dimensions such that

$$
\begin{aligned}
\Phi_{k i}^{-}= & \left(\begin{array}{cccc}
\phi_{11}^{k i-} & \phi_{12}^{k i} & P_{k i} & \tau A^{\top} Z_{k i} \\
* & \phi_{22}^{k i} & 0 & \tau \lambda_{k i} \Gamma_{k}^{\top} Z_{k i} \\
* & * & -\varepsilon I_{n} & \tau Z_{k i} \\
* & * & * & -\tau Z_{k i}
\end{array}\right)<0, \\
& i \in \mathcal{N}_{1}, \quad k \in \mathcal{M}^{-}, \\
\Phi_{k i}^{+}= & \left(\begin{array}{cccc}
\phi_{11}^{k i+} & \phi_{12}^{k i} & P_{k i} & \tau A^{\top} Z_{k i} \\
* & \phi_{22}^{k i+} & 0 & \tau \lambda_{k i} \Gamma_{k}^{\top} Z_{k i} \\
* & * & -\varepsilon I_{n} & \tau Z_{k i} \\
* & * & * & -\tau Z_{k i}
\end{array}\right)<0, \\
& i \in \mathcal{N}_{1}, \quad k \in \mathcal{M}^{+}, \\
\Theta_{k i}= & \left(\begin{array}{ccc}
X_{11}^{k i} & X_{12}^{k i} & Y_{k i} \\
* & X_{22}^{k i} & T_{k i} \\
* & * & \mathrm{e}^{-\tilde{\alpha} \tau} Z_{k i}
\end{array}\right) \geq 0 \\
& i \in \mathcal{N}_{1}, \quad k \in \mathcal{M}
\end{aligned}
$$

where

$$
\begin{aligned}
\phi_{11}^{k i-}= & P_{k i} A+A^{\top} P_{k i}+Y_{k i}^{\top}+Y_{k i}+Q_{k i}+\tau X_{11}^{k i}+\varepsilon \gamma^{2} I_{n} \\
& +\alpha^{-} P_{k i}, \\
\phi_{22}^{k i-}= & -T_{k i}-T_{k i}^{\top}-(1-d) \mathrm{e}^{-\alpha^{-} \tau} Q_{k i}+\tau X_{22}^{k i}, \\
\phi_{11}^{k i+}= & P_{k i} A+A^{\top} P_{k i}+Y_{k i}^{\top}+Y_{k i}+Q_{k i}+\tau X_{11}^{k i}+\varepsilon \gamma^{2} I_{n} \\
& -\alpha^{+} P_{k i}, \\
\phi_{22}^{k i+}= & -T_{k i}-T_{k i}^{\top}-(1-d) Q_{k i}+\tau X_{22}^{k i}
\end{aligned}
$$

and $\tilde{\alpha}=\alpha^{-}$for $k \in \mathcal{M}^{-}$, otherwise $\tilde{\alpha}=0$. Then the synchronization manifold $\mathbb{S}$ is globally exponentially stable for any switching signal satisfying the condition (29) and the average dwell time

$$
T_{a} \geq T_{a}^{*}=\frac{\ln \mu}{\alpha^{*}-\alpha} .
$$

where $\alpha^{*}, \alpha, \mu$ and $\phi_{12}^{k}$ are defined as in Theorem 2, but with $Z_{k 1}=\mathbf{0} \in \mathbb{R}^{n \times n}$.

Proof: Select the piecewise Lyapunov functional (17) and (18) when $\sigma(t)=k \in \mathcal{M}^{-}$, and (34) for $\sigma(t)=k \in \mathcal{M}^{+}$. Combining the proof of Theorem 2 and Theorem 3, gives the proof of Theorem 4 directly. Thus, it is omitted here.
Remark 11: In Theorem 1 and Theorem 2, the obtained LMIs (13) and (14) and (30), (31), and (32) are completely decoupled from each other for the index $i=2, \ldots, N$. This is to say that we can solve $2 m(N-1)$ and $3 m(N-1)$ independently relatively lower dimensional LMIs to analyze the synchronizability of the entire switched network, no matter how many nodes the network has. So there is no obstacle to apply these results to a large scale network. On the other hand, for the global synchronization counter part, due to the existence of the nonlinear term $f(\cdot)$ in the node dynamics (38), the LMIs obtained in Theorem 3 and Theorem 4 are related by a positive scaler $\varepsilon$ for the index $i$. This will influence the application of those results to large scale networks somehow.

\section{Simulations}

In this section, we will give three different examples to illustrate the main results obtained above, including a network using the Lyapunov stable linear system as its nodes, a communication network with mobile agents, and a network consisting of coupled chaotic systems. For simplicity, we assume that there are only two subnetworks in all examples, i.e., $\mathcal{M}=\{1,2\}$, and each subnetwork has 10 identical nodes, ie., $N=10$. We also assume that $d(t)=0.005(1-\sin (t))$. So $\tau=0.01$ and $d=0.005$ make the Assumption 1 hold.

Example 1: Consider a network (1) with each node being a two-dimensional linear system. The isolate dynamics is described by

$$
\dot{x}=A x=\left(\begin{array}{cc}
0 & -0.5 \\
0.5 & 0
\end{array}\right) x .
$$

The system (59) is stable, but not asymptotically stable with both eigenvalues $\pm 0.5 i$ of $A$ on the imaginary axis. Suppose the outer coupling matrices $C_{1}, C_{2}$ are given below

$$
\begin{gathered}
C_{1}=\left(\begin{array}{cccccccccc}
-4 & 1 & 1 & 0 & 0 & 0 & 0 & 0 & 1 & 1 \\
1 & -4 & 1 & 1 & 0 & 0 & 0 & 0 & 0 & 1 \\
1 & 1 & -4 & 1 & 1 & 0 & 0 & 0 & 0 & 0 \\
0 & 1 & 1 & -4 & 1 & 1 & 0 & 0 & 0 & 0 \\
0 & 0 & 1 & 1 & -4 & 1 & 1 & 0 & 0 & 0 \\
0 & 0 & 0 & 1 & 1 & -4 & 1 & 1 & 0 & 0 \\
0 & 0 & 0 & 0 & 1 & 1 & -4 & 1 & 1 & 0 \\
0 & 0 & 0 & 0 & 0 & 1 & 1 & -4 & 1 & 1 \\
1 & 0 & 0 & 0 & 0 & 0 & 1 & 1 & -4 & 1 \\
1 & 1 & 0 & 0 & 0 & 0 & 0 & 1 & 1 & -4
\end{array}\right) \\
C_{2}=\left(\begin{array}{ccccccccccc}
-4 & 1 & 0 & 0 & 1 & 0 & 0 & 1 & 0 & 1 \\
1 & -2 & 1 & 0 & 0 & 0 & 0 & 0 & 0 & 0 \\
0 & 1 & -3 & 1 & 0 & 0 & 0 & 1 & 0 & 0 \\
0 & 0 & 1 & -3 & 1 & 0 & 1 & 0 & 0 & 0 \\
1 & 0 & 0 & 1 & -3 & 1 & 0 & 0 & 0 & 0 \\
0 & 0 & 0 & 0 & 1 & -3 & 1 & 0 & 0 & 1 \\
0 & 0 & 0 & 1 & 0 & 1 & -3 & 1 & 0 & 0 \\
1 & 0 & 1 & 0 & 0 & 0 & 1 & -4 & 1 & 0 \\
0 & 0 & 0 & 0 & 0 & 0 & 0 & 1 & -2 & 1 \\
1 & 0 & 0 & 0 & 0 & 1 & 0 & 0 & 1 & -3
\end{array}\right) .
\end{gathered}
$$

Since $C_{1}$ and $C_{2}$ are symmetric matrices, then there exist two unitary matrices $U_{1}$ and $U_{2}$ such that $U_{1}^{\top} C_{1} U_{1}=\Lambda_{1}=$ $\operatorname{diag}\{0,-1.76,-1.76,-4,-5,-5,-5,-5,-6.23,-6.23\}$ and $U_{2}^{\top} C_{2} U_{2}=\Lambda_{2}=\operatorname{diag}\{0,-1.19,-1.38,-2$, $-2.8,-3.38,-3.62,-3.86,-5.62,-6.15\}$. It is easy to 


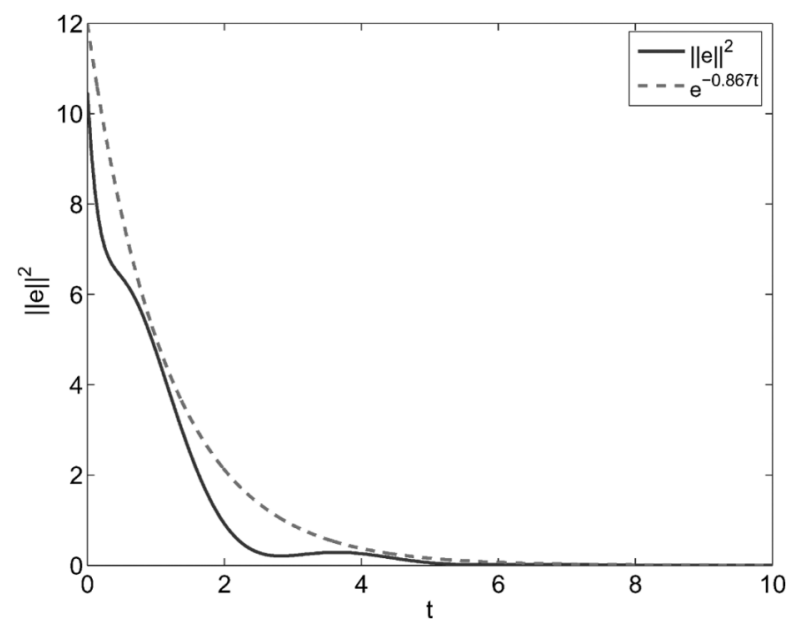

Fig. 1. The synchronization error $\|e\|^{2}$ and the decay rate bound of the subnetwork with $\left\{C_{1}, \Gamma_{1}\right\}$.

verify that $U_{1} \neq U_{2}$. Assume that the inner coupling matrices are

$$
\Gamma_{1}=\left(\begin{array}{cc}
0.25 & 0 \\
-1 & 0.25
\end{array}\right), \quad \Gamma_{2}=\left(\begin{array}{cc}
0.5 & 2 \\
-0.1 & 0.5
\end{array}\right)
$$

By solving LMIs (13) and (14) repeatedly with different values of $\alpha$, we see that the two subnetworks with coupling matrices $\left\{C_{1}, \Gamma_{1}\right\}$ and $\left\{C_{2}, \Gamma_{2}\right\}$ are both exponentially self-synchronizing, and the best decay rate bounds we can get are $\alpha_{1}=0.867$ and $\alpha_{2}=1.162$, respectively. Choosing $\alpha=\min \left\{\alpha_{1}, \alpha_{2}\right\}=0.867$, and solving the LMIs (13) and (14), gives the corresponding matrices $P_{k i}, Q_{k i}$ and $Z_{k i}$ with $k=1,2$ and $i=2,3, \ldots, 10$. Thus, we can get $\mu=4.072$ and $T_{a}^{*}=\ln \mu / \alpha=1.6195$. According to Theorem 1, the synchronization manifold of the switched network is exponentially stable under the average dwell time switching law with $T_{a}>T_{a}^{*}$.

Figs. 1-3 show the time evolution of the synchronization errors $\|e\|^{2}$ and the decay rate estimations of the two subnetworks and the switched network with $T_{a}=1.8195$, respectively. From Figs. 1 and 2, we see that the convergence rate estimations of the two subnetworks are quite good. Moreover, the estimated decay rate of the switched network is $\alpha-\ln \mu / T_{a}=0.1$. However, Fig. 3 shows that the decay rate of the overall network is at least 0.6. It is to say that this estimation is not as good as the ones of the subnetworks, and the conservativeness for the switched network's decay rate is relatively large as we discussed in Remark 5.

Compared to our estimation $T_{a}^{*}=1.6195$ obtained from Theorem 1, simulation shows that the switched network with node (59) can still achieve synchronization under the average dwell time switching signal with $T_{a}^{*} \approx 1.275$. So the theoretical bound proposed in the paper has some conservativeness. However, as we discussed in Remark 6, those theoretical estimations give an safety guide in practice.

Figs. 4 and 6 are the state responses of the switched network under different switching signals $\sigma(t)$ with $T_{a}=1.8195$ and $T_{a}=1$, which demonstrate that the average dwell time switching scheme can guarantee synchronizability of the overall switched networks, and meanwhile the relatively fast switching will destroy the synchronizability, even when the switching happens between two exponential synchronizable subnetworks. The corresponding switching signals are drawn in Fig. 5 and Fig. 7.

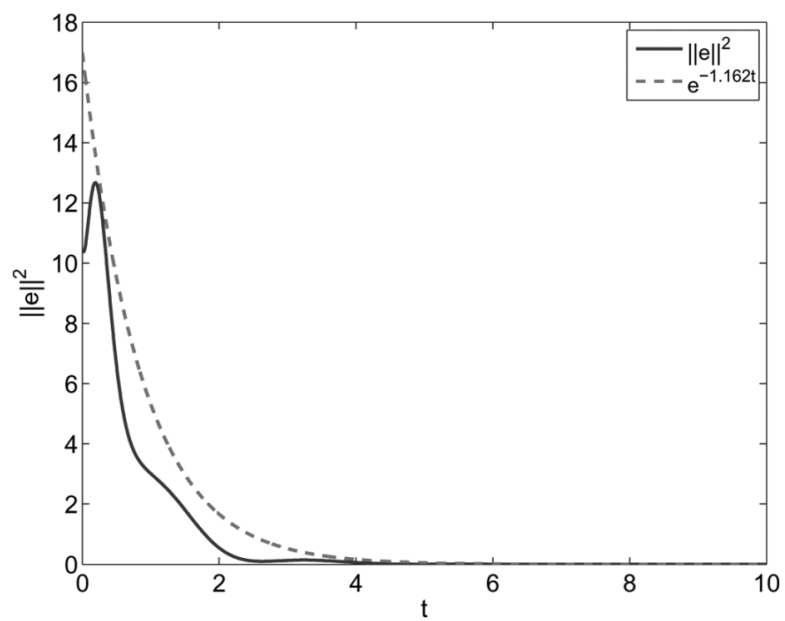

Fig. 2. The synchronization error $\|e\|^{2}$ and the decay rate bound of the subnetwork with $\left\{C_{2}, \Gamma_{2}\right\}$.

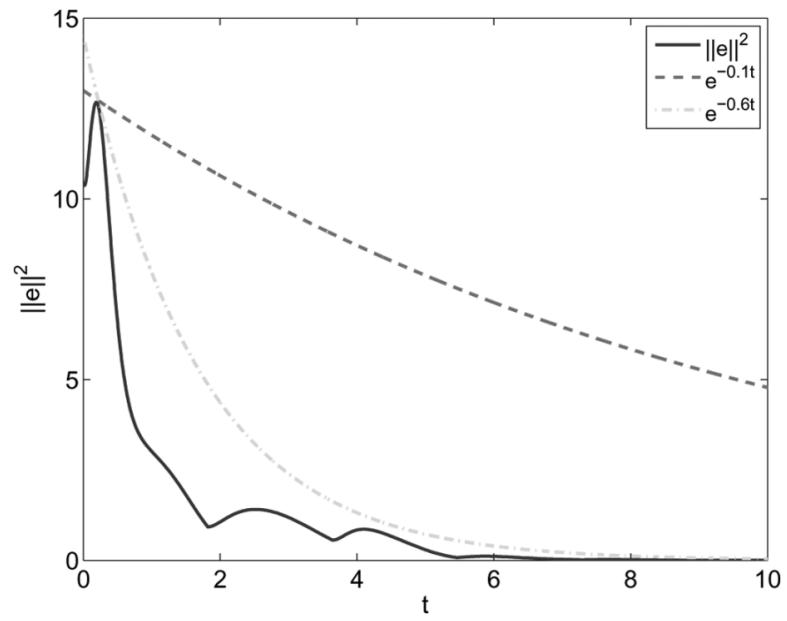

Fig. 3. The synchronization error $\|e\|^{2}$ and the decay rate bound of the switched network with $T_{a}=1.8195$.

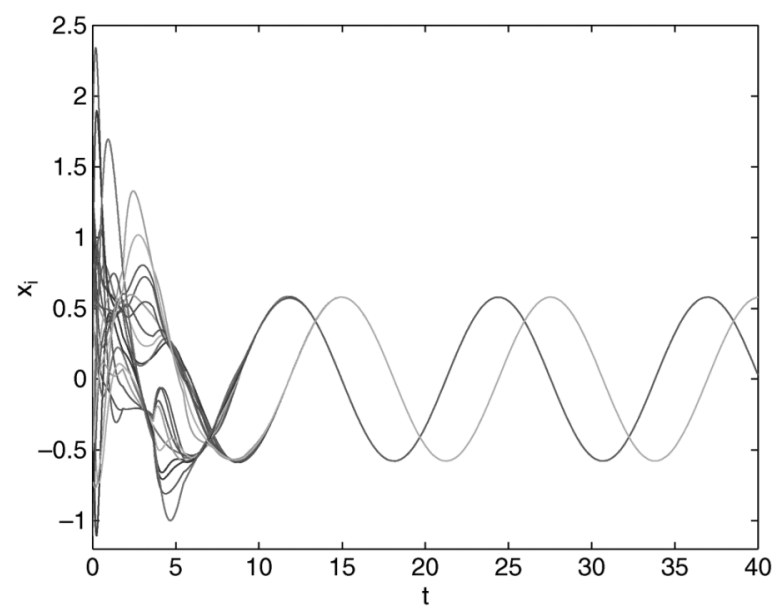

Fig. 4. The state responses $x_{i}$ of the switched network with $T_{a}=1.8195$.

Example 2: The consensus problems of a multiagent system have important practical applications including cooperative control of unmanned air vehicles, formation control of mobile robots, control of communication networks, and flocking of social insects [23]. Now we will try to use our results to analyze consensus problems.

Consider a second order multiagent system, its $i$ th agent has the dynamics as follows [44]: 


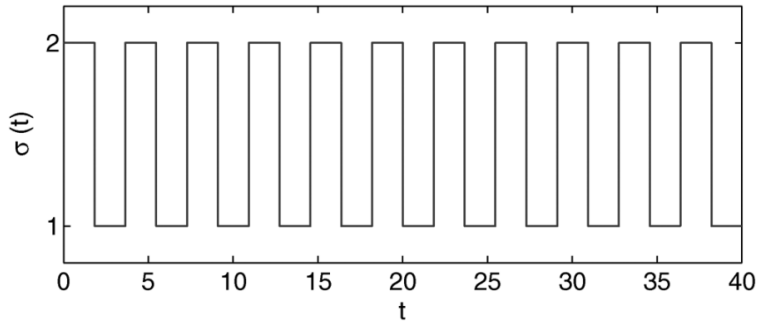

Fig. 5. The switching signal $\sigma(t)$ with $T_{a}=1.8195$.

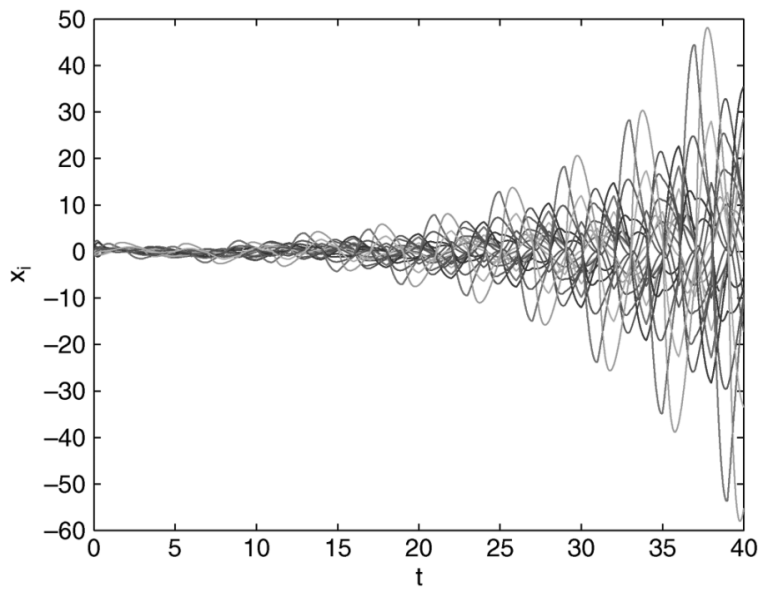

Fig. 6. The state responses $x_{i}$ of the switched network with $T_{a}=1$.

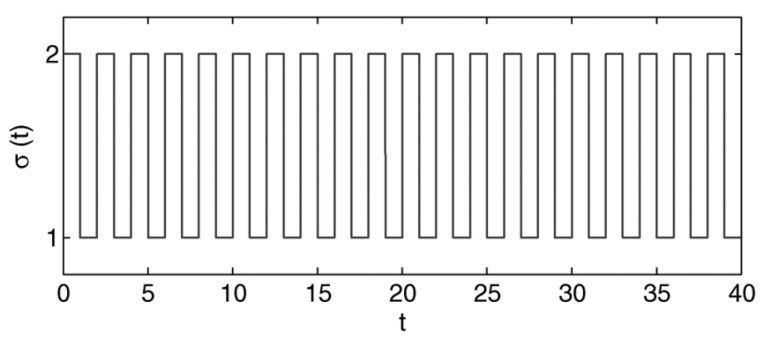

Fig. 7. The switching signal $\sigma(t)$ with $T_{a}=1$.

$$
\begin{aligned}
& \dot{p}_{i}(t)=v_{i}(t) \\
& \dot{v}_{i}(t)=u_{i}(t)
\end{aligned}
$$

where $p_{i}(t) \in \mathbb{R}$ is the position state, $v_{i}(t) \in \mathbb{R}$ is the velocity state, and $u_{i}(t) \in \mathbb{R}$ is the protocol. In [44], the linear consensus protocol with constant time delay is used. Here we consider the protocol with time-varying communication delay, which is described as

$$
u_{i}(t)=-a v_{i}(t)+\sum_{j=1}^{N} c_{i j}^{\sigma(t)}\left(p_{j}(t-d(t))-p_{i}(t-d(t))\right) .
$$

The topology switching is due to link failure or creation when agents are moving [23].

It says that the consensus problem of multiagent system (60) is solved if the states of the agents satisfy $\lim _{t \rightarrow \infty}\left(p_{i}(t)-\right.$ $\left.p_{j}(t)\right)=0$, and $\lim _{t \rightarrow \infty} v_{i}(t)=0, \forall i, j \in \mathcal{N}$ [44].
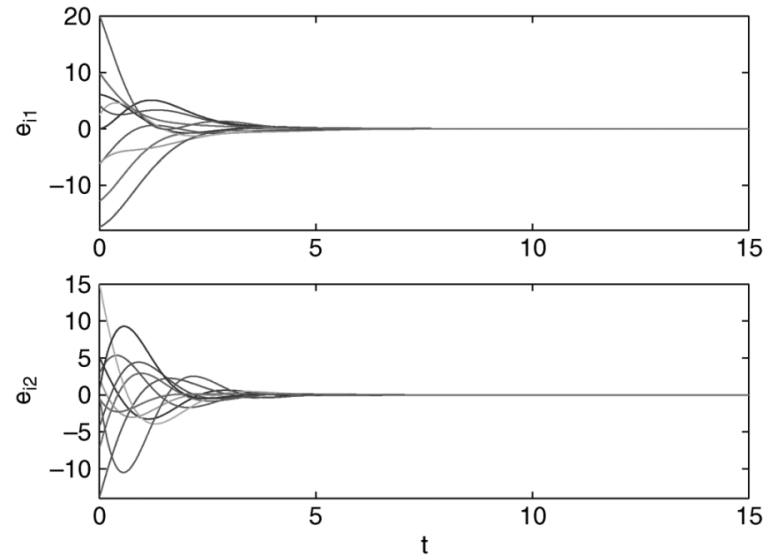

Fig. 8. The synchronization errors $e_{i}$ of the self-synchronizing subnetwork.
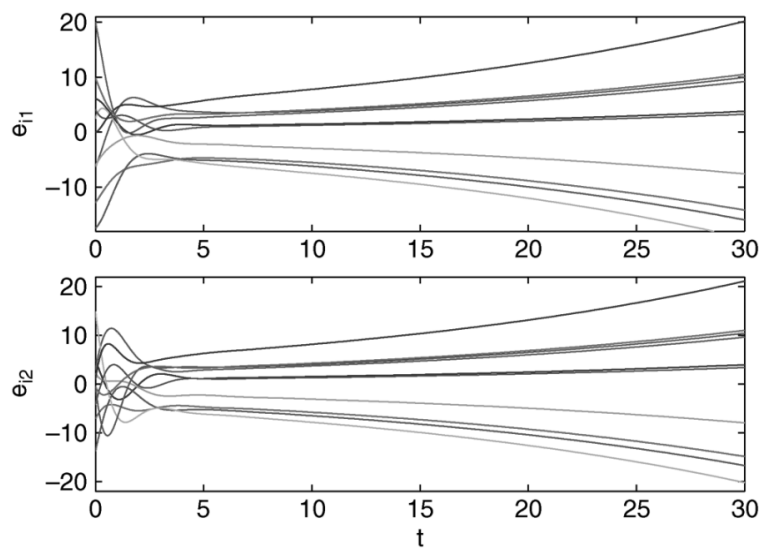

Fig. 9. The synchronization errors $e_{i}$ of the nonsynchronizing subnetwork.

Similarly as in [44], let $x_{i 1}=2 v_{i} / a+p_{i}, x_{i 2}=v_{i}, x_{i}=$ $\left(x_{i 1}, x_{i 2}\right)^{\top}$ and $x=\left(x_{1}^{\top}, x_{2}^{\top}, \ldots, x_{N}^{\top}\right)^{\top}$, one can get

$$
\dot{x}=\left(I_{N} \otimes A\right) x+\left(C_{\sigma(t)} \otimes \Gamma\right) x(t-d(t))
$$

where

$$
A=\left(\begin{array}{cc}
-\frac{a}{2} & \frac{a}{2} \\
\frac{a}{2} & -\frac{a}{2}
\end{array}\right), \text { and } \Gamma=\left(\begin{array}{cc}
0 & 0 \\
\frac{2}{a} & 0
\end{array}\right) .
$$

It is obvious that System (62) can be seen as a special case of our model (1) with linear node dynamics, and consensus problem of (62) can also be seen as a synchronization problem. Here, for simplicity, only two subnetworks are considered, the situation with more subnetworks can be analyzed similarly. Suppose $a=2 . C_{1}$ and $C_{2}$ are given as

$$
C_{1}=\left(\begin{array}{cccccccccc}
-4 & 1 & 1 & 0 & 0 & 0 & 0 & 0 & 1 & 1 \\
1 & -4 & 1 & 1 & 0 & 0 & 0 & 0 & 0 & 1 \\
1 & 1 & -4 & 1 & 1 & 0 & 0 & 0 & 0 & 0 \\
0 & 1 & 1 & -2 & 0 & 0 & 0 & 0 & 0 & 0 \\
0 & 0 & 1 & 0 & -2 & 1 & 0 & 0 & 0 & 0 \\
0 & 0 & 0 & 0 & 1 & -2 & 1 & 0 & 0 & 0 \\
0 & 0 & 0 & 0 & 0 & 1 & -3 & 1 & 1 & 0 \\
0 & 0 & 0 & 0 & 0 & 0 & 1 & -3 & 1 & 1 \\
1 & 0 & 0 & 0 & 0 & 0 & 1 & 1 & -4 & 1 \\
1 & 1 & 0 & 0 & 0 & 0 & 0 & 1 & 1 & -4
\end{array}\right)
$$



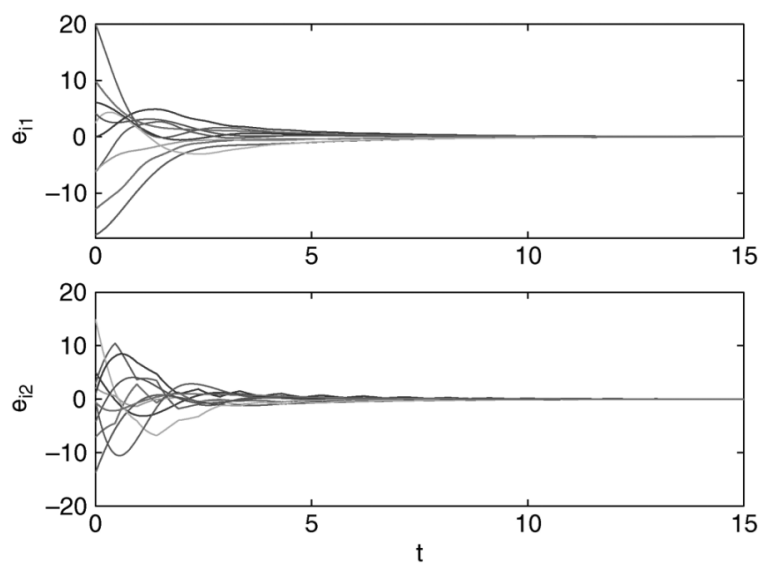

Fig. 10. The synchronization errors $e_{i}$ of the switched network.

$$
C_{2}=\left(\begin{array}{cccccccccc}
-4 & 1 & 1 & 0 & 0 & 0 & 0 & 0 & 1 & 1 \\
1 & -2 & -1 & 1 & 0 & 0 & 0 & 0 & 0 & 1 \\
1 & -1 & -2 & 1 & 1 & 0 & 0 & 0 & 0 & 0 \\
0 & 1 & 1 & -3 & 0 & 1 & 0 & 0 & 0 & 0 \\
0 & 0 & 1 & 0 & -2 & 1 & 0 & 0 & 0 & 0 \\
0 & 0 & 0 & 1 & 1 & -2 & 1 & -1 & 0 & 0 \\
0 & 0 & 0 & 0 & 0 & 1 & -3 & 1 & 1 & 0 \\
0 & 0 & 0 & 0 & 0 & -1 & 1 & -2 & 1 & 1 \\
1 & 0 & 0 & 0 & 0 & 0 & 1 & 1 & -4 & 1 \\
1 & 1 & 0 & 0 & 0 & 0 & 0 & 1 & 1 & -4
\end{array}\right)
$$

Apparently, for the given subnetworks, the one with $\left\{C_{1}, \Gamma\right\}$ is self-synchronizing, and the one with $\left\{C_{2}, \Gamma\right\}$ is nonsynchronizing. See Figs. 8 and 9 for the synchronization errors of each subnetwork. It is worth to mention that $C_{2}$ has four negative off-diagonal entries $c_{23}^{2}=c_{32}^{2}=-1$ and $c_{68}^{2}=c_{86}^{2}=-1$. These negative connections may be caused by the disturbance, environment noise or sensor failure of the corresponding agents. And these negative entries make $C_{2}$ have a positive eigenvalue $\lambda=0.0971$. As we discussed in Remark 4, our analysis method is still applicable to this kind of network. Solving LMIs (30), (31), and (32) in Theorem 2 gives the associated positive matrices $P_{k i}, Q_{k i}$ and $Z_{k i}$ with $\alpha^{-}=0.85, \alpha^{+}=0.1$. Then one obtains $\mu=1.2$. Let $\alpha^{*}=0.4$. Theorem 2 shows that the network (62) is exponentially synchronized if the switching signal $\sigma(t)$ satisfy

$$
\inf _{t \geq t_{0}} \frac{T^{-}(t)}{T^{+}(t)} \geq \frac{\alpha^{+}+\alpha^{*}}{\alpha^{-}-\alpha^{*}}=1.1111
$$

and the average dwell time

$$
T_{a}>T_{a}^{*}=\frac{\ln \mu}{\alpha^{*}}=0.4558 .
$$

Fig. 10 gives the synchronization errors of the switched network with a switching signal satisfying (63) and (64) which is shown in Fig. 11 with $T_{a}=0.456$ and the activation time of the self-synchronizing and nonsynchronizing subnetwork being $T_{a}$ and $1.111 T_{a}$, respectively. Obviously, all the synchronization errors do exponentially converge to zero, i.e., the consensus problem of the multiagent system (60) is achieved exponentially.

Example 3: In the above two examples, only linear systems are considered as the node dynamics. Now let's consider a more complicated network which consists of interconnected modified

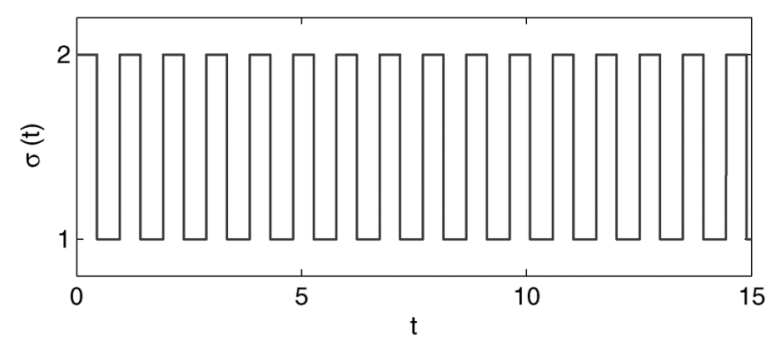

Fig. 11. The switching signal $\sigma(t)$ with $T_{a}=0.456$.

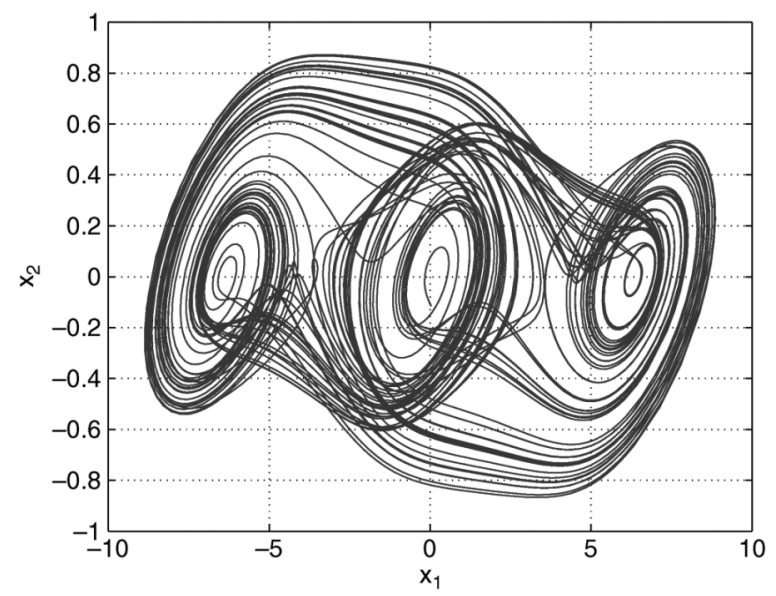

Fig. 12. Chaotic three-scroll attractors on the $x_{1}-x_{2}$ plane.

Chua's circuit. The single modified Chua's circuit is described by [45] and [46]

$$
\dot{x}=A x+f(x)=\left(\begin{array}{ccc}
0 & \rho & 0 \\
1 & -1 & 1 \\
0 & -\beta & 0
\end{array}\right) x+\left(\begin{array}{c}
-\rho f_{1}\left(x_{1}\right) \\
0 \\
0
\end{array}\right)
$$

where

$$
f_{1}\left(x_{1}\right)= \begin{cases}\frac{b \pi}{2 a}\left(x_{1}-2 a c_{1}\right), & x_{1} \geq 2 a c_{1}, \\ -b \sin \left(\frac{\pi x_{1}}{2 a}+\pi\right), & \left|x_{1}\right|<2 a c_{1}, \\ \frac{b \pi}{2 a}\left(x_{1}+2 a c_{1}\right), & x_{1} \leq-2 a c_{1}\end{cases}
$$

in which $\rho>0, \beta>0, a>0, b>0$. In this example, we set the parameters $\rho=9.5, \beta=11, a=1.6, b=0.1, c=2$, and $d_{1}=\pi$. From [46], it is directly to have that the modified Chua's circuit with the given parameters is a chaotic system which has a 3-scroll attractor depicted in Fig. 12.

Suppose the inner coupling matrices $\Gamma_{1}=\Gamma_{2}=\Gamma=$ $\operatorname{diag}\{1.5,1,1\}$, and the outer coupling $C_{1}$ is the same as in Example 1, and $C_{2}$ is given as follows:

$$
C_{2}=\left(\begin{array}{cccccccccc}
-1 & 0 & 1 & 0 & 0 & 0 & 0 & 0 & 0 & 0 \\
0 & -1 & 0 & 1 & 0 & 0 & 0 & 0 & 0 & 0 \\
1 & 0 & -2 & 1 & 0 & 0 & 0 & 0 & 0 & 0 \\
0 & 1 & 1 & -3 & 0 & 1 & 0 & 0 & 0 & 0 \\
0 & 0 & 0 & 0 & -1 & 1 & 0 & 0 & 0 & 0 \\
0 & 0 & 0 & 1 & 1 & -4 & 1 & 1 & 0 & 0 \\
0 & 0 & 0 & 0 & 0 & 1 & -3 & 1 & 1 & 0 \\
0 & 0 & 0 & 0 & 0 & 1 & 1 & -4 & 1 & 1 \\
0 & 0 & 0 & 0 & 0 & 0 & 1 & 1 & -3 & 1 \\
0 & 0 & 0 & 0 & 0 & 0 & 0 & 1 & 1 & -2
\end{array}\right) .
$$

It is easy to get that condition (40) is satisfied, and the subnetwork $\left\{C_{1}, \Gamma\right\}$ is self-synchronizing, but the one $\left\{C_{2}, \Gamma\right\}$ is nonsynchronizing. Figs. 13 and 14 show the synchronization errors of the subnetworks. Selecting $\alpha^{-}=1.44, \alpha^{+}=3.4$ 

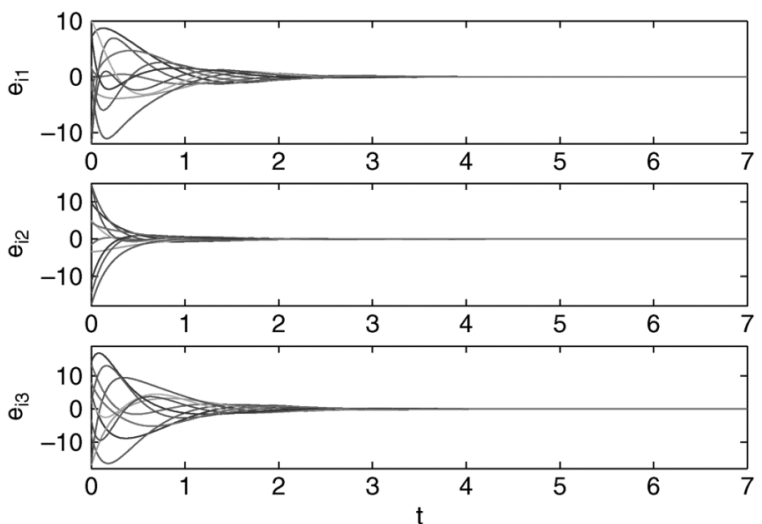

Fig. 13. The synchronization errors $e_{i}$ of the self-synchronizing subnetwork.
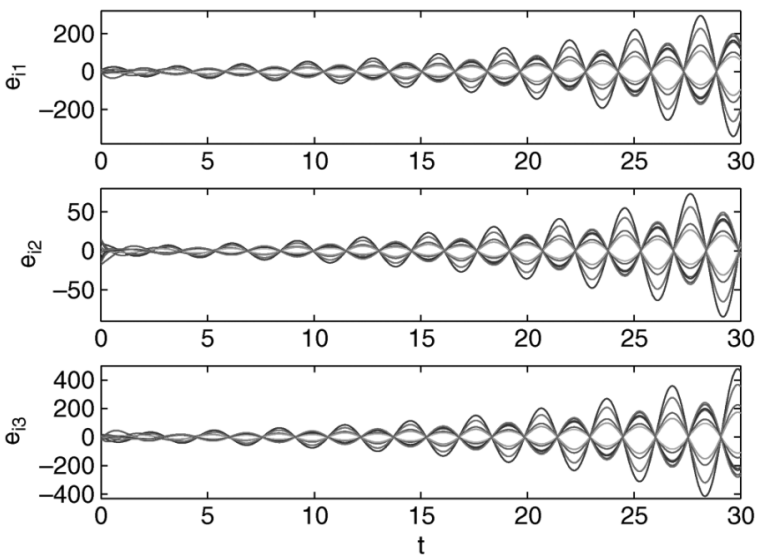

Fig. 14. The synchronization errors $e_{i}$ of the nonsynchronizing subnetwork.
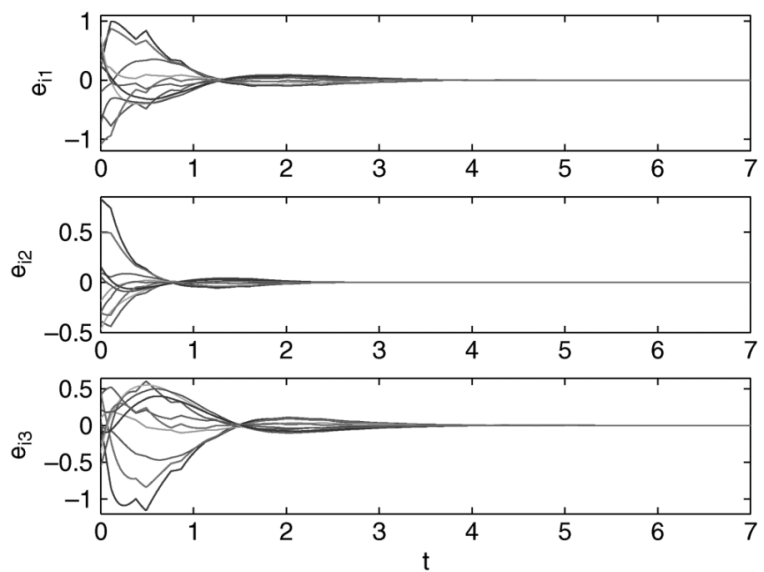

Fig. 15. The synchronization errors $e_{i}$ of the switched network.

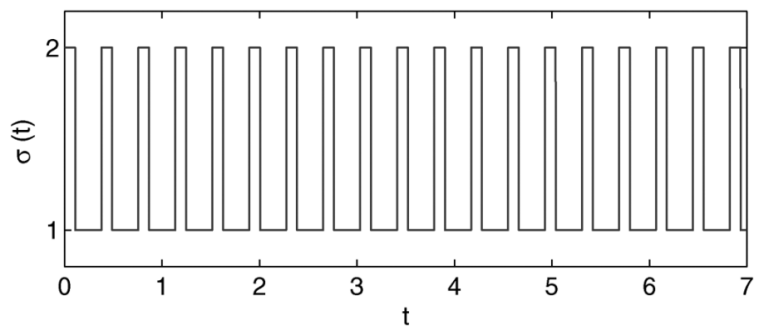

Fig. 16. The switching signal $\sigma(t)$ with $T_{a}=0.11$.

and solving LMIs (55), (56) and (57) in Theorem 4, we can get the associated positive matrices $P_{k i}, Q_{k i}$ and $Z_{k i}$ to compute $\mu=1.001$. Let $\alpha^{*}=0.1$. According to Theorem 4, the network
(38) with modified Chua's circuit as its nodes is exponentially synchronized under the switching signal $\sigma(t)$ satisfying

$$
\inf _{t \geq t_{0}} \frac{T^{-}(t)}{T^{+}(t)} \geq \frac{\alpha^{+}+\alpha^{*}}{\alpha^{-}-\alpha^{*}}=2.4476
$$

and the average dwell time

$$
T_{a}>T_{a}^{*}=\frac{\ln \mu}{\alpha^{*}}=0.1 .
$$

Fig. 15 gives the synchronization errors of the switched network with a specified switching signal satisfying (65) and (66). The switching signal is shown in Fig. 16, where $T_{a}=0.11$ and the activation time of the self-synchronizing and nonsynchronizing subnetwork are $T_{a}$ and $2.4476 T_{a}$. Thus, we have that all the synchronization errors exponentially converge to zero.

\section{CONCLUSION}

In this paper, we presented a new model of a complex delayed dynamical network with switching topology where the coupling delays are time-varying. The problem of local and global exponential synchronization of a class of such switched networks has been investigated. By using the average dwell time method in switched systems theory, for both cases: i) all subnetworks are self-synchronizing and ii) not all subnetworks are self-synchronizing, we have obtained delay-dependent sufficient conditions in terms of LMIs and identified switching signals under which the network is locally exponentially synchronized. We also discussed the global synchronization of the network which has a special isolate dynamics. Similar to the local results, global conditions were obtained for both of the cases. Finally, we gave simulation examples of delayed networks with each example having two couple of different coupling matrices to demonstrate the effective of the proposed results.

\section{REFERENCES}

[1] S. Boccaletti, V. Latora, Y. Moreno, M. Chavez, and D. U. Hwang, "Complex networks: Structure and dynamics," Phys. Rep., vol. 424, pp. 175-308, 2006.

[2] M. Newman, "The structure and functions of complex networks," SIAM Rev., vol. 45, pp. 167-256, 2003.

[3] X. F. Wang and G. Chen, "Complex networks: Small-world, scale-free, and beyond," IEEE Circuits Syst. Mag., vol. 3, no. 1, pp. 6-20, 2003.

[4] K. Kaneko, "Coupled map lattices," Chaos, vol. 2, no. 3, pp. 279-282, 1992.

[5] C. W. Wu and L. O. Chua, "Synchronization in an array of linearly coupled dynamical systems," IEEE Trans. Circuits Syst. I, Fundam. Theory Appl., vol. 42, no. 8, pp. 430-447, Aug. 1995.

[6] L. Pecora and T. Carroll, "Master stability function for synchronized coupled systems," Phys. Rev. Lett., vol. 80, pp. 2109-2112, 1998.

[7] J. Lü and G. Chen, "A time-varying complex dynamical network model and its controlled synchronization criteria," IEEE Trans Autom. Control., vol. 50, no. 6, pp. 841-846, Jun. 2005.

[8] C. Zhou and J. Kurths, "Dynamical networks and enhanced synchronization in adaptive complex networks," Phys. Rev. Lett., vol. 96, p. $164102,2006$.

[9] M. Chen, "Chaos synchronization in complex networks," IEEE Trans. Circuits Syst. I, Reg. Papers, vol. 55, no. 5, pp. 1335-1346, Jun. 2008.

[10] C. Li and G. Chen, "Synchronization in general complex dynamical networks with coupling delays," Phys. A, vol. 343, pp. 263-278, 2004.

[11] J. Zhou and T. Chen, "Synchronization in general complex delayed dynamical networks," IEEE Trans. Circuits Syst. I, Reg. Papers, vol. 53, no. 3, pp. 733-744, Mar. 2006.

[12] J. Lu and D. W. C. Ho, "Local and global synchronization in general complex dynamical networks with delay coupling," Chaos, Solitons Fractals, vol. 37, no. 5, pp. 1497-1510, 2008.

[13] H. Gao, J. Lam, and G. Chen, "New criteria for synchronization stability of general complex dynamical networks with coupling delays," Phys. Lett. A, vol. 360, pp. 263-273, 2006. 
[14] B. Liu and H. J. Marquez, "Uniform stability of discrete delay systems and synchronization of discrete delay dynamical networks via Razumikhin technique," IEEE Trans. Circuits Syst. I, Reg. Papers, vol. 55, no. 9, pp. 2795-2805, Sep. 2008.

[15] A. E. Motter, C. S. Zhou, and J. Kurths, "Enhancing complex network synchronization," Europhys. Lett., vol. 69, no. 3, pp. 334-340, 2005.

[16] M. Chavez, D. U. Hwang, A. Amann, H. Hentschel, and S. Boccaletti, "Synchronization is enhanced in weighted complex networks," Phys. Rev. Lett., vol. 94, p. 218701, 2005.

[17] T. Liu, J. Zhao, and D. J. Hill, "Synchronization of complex delayed dynamical networks with nonlinearly coupled nodes," Chaos Solitons Fractals, vol. 40, pp. 1506-1519, 2009.

[18] M. Barahona and L. M. Pecora, "Synchronization in small-world systems," Phys. Rev. Lett., vol. 89, p. 054101, 2002.

[19] T. Nishikawa and A. E. Motter, "Maximum performance at minimum cost in network synchronization," Physica D, vol. 224, pp. 77-89, 2006.

[20] G. Chen and Z. Duan, "Network synchronizability analysis: A graphtheoretic approach," Chaos, vol. 18, p. 037102, 2008.

[21] A. Arenas, A. Diaz-Guilera, J. Kurths, Y. Moreno, and C. Zhou, "Synchronization in complex networks," Phys. Rep., vol. 469, pp. 93-153, 2008.

[22] C. W. Wu, Synchronization in Complex Networks of Nonlinear Dynamical Systems. Singapore: World Scientific, 2007.

[23] R. Olfati-Saber and R. M. Murray, "Consensus problems in networks of agents with switching topology and time-delays," IEEE Trans Autom. Control, vol. 49, no. 9, pp. 1520-1533, Sep. 2004.

[24] I. V. Belykh, V. N. Belykh, and M. Hasler, "Blinking model and synchronization in small-world networks with a time-varying coupling," Phys. D, vol. 195, pp. 188-206, 2004.

[25] D. J. Stilwell, E. M. Bollt, and D. G. Roberson, "Sufficient conditions for fast switching synchronization in time-varying network topologies," SIAM J. Applied Dynam. Syst., vol. 5, no. 1, pp. 140-156, 2006.

[26] J. Yao, D. J. Hill, Z. H. Guan, and H. O. Wang, "Synchronization of complex dynamical networks with switching topology via adaptive control," in Proc. 45th IEEE CDC, 2006, pp. 2819-2824.

[27] J. Zhao, D. J. Hill, and T. Liu, "Synchronization of complex dynamical networks with switching topology: A switched system point of view," Automatica, vol. 45, no. 11, pp. 2502-2511, 2009.

[28] S. Kim and D. J. Hill, "Synchronization of a complex network with switched coupling," in Proc 17th World Congr., Seoul, Korea, 2008, pp. 1559-1564.

[29] M. Porfiri, D. J. Stilwell, and E. M. Bollt, "Synchronization in random weighted directed networks," IEEE Trans. Circuits Syst. I, Reg. Papers, vol. 55 , no. 10 , pp. $3170-3177$, Oct. 2008.

[30] T. Liu and J. Zhao, "Synchronization of complex switched delay dynamical networks with simultaneously diagonalizable coupling matrices," J. Control Theory Appl., vol. 6, no. 4, pp. 351-356, 2008.

[31] R. E. Amritkar and C. K. Hu, "Synchronized state of coupled dynamics on time-varying networks," Chaos, vol. 16, p. 015177, 2006.

[32] L. Chen, C. Qiu, and H. B. Huang, "Synchronization with no-off coupling: Role of time scales in network dynamics," Phys. Rev. E, vol. 79, p. 045101, 2009.

[33] W. L. Lu, F. M. Atay, and J. Jost, "Chaos synchronization in networks with coupled maps with time-varying topologies," Eur. Phys. J. B, vol. 63, pp. 399-406, 2008.

[34] W. L. Lu, F. M. Atay, and J. Jost, "Synchronization of discrete-time dynamical networks with time-varying couplings," SIAM J. Math. Anal., vol. 39, no. 4, pp. 1231-1259, 2007.

[35] M. Porfiri and F. Fiorilli, "Node-to-node pinning control of complex networks," Chaos, vol. 19, p. 013122, 2009.

[36] M. Porfiri, D. J. Stilwell, E. M. Bollt, and J. D. Skufca, "Random talk: Random walk and synchronizability in a moving neighborhood network," Phys. D, vol. 224, pp. 102-113, 2006.

[37] W. Xia and J. Cao, "Pinning synchronization of delayed dynamical networks via periodically intermittent control," Chaos, vol. 19, p. 013120, 2009.

[38] D. Liberzon, Switching in Systems and Control. Boston, MA: Birkhauser, 2003.

[39] J. P. Hespanha and A. S. Morse, "Stability of switched systems with average dwell-time," in 38th IEEE Conf. Decision Control, 1999, pp. 2655-2660.

[40] X. M. Sun, J. Zhao, and D. J. Hill, "Stability and L2-gain analysis for switched delay systems: A delay-dependent method," Automatica, vol. 42, pp. 1769-1774, 2006

[41] G. Zhai, B. Hu, K. Yasuda, and A. N. Michel, "Piecewise Lyapunov function for switched systems with average dwell time," Asian J. Control, vol. 2, no. 3, pp. 192-197, 2000.

[42] J. P. Hespanha, "Uniform stability of switched linear systems: Extensions of LaSalle's invariance principle," IEEE Trans. Autom. Control, vol. 49, no. 3, pp. 470-482, Mar. 2004.
[43] S. Boyd, L. E. Ghaoui, E. Feron, and V. Balakrishnan, Linear Matrix Inequalities in System and Control Theory. Philadelphia, PA: SIAM, 1994.

[44] P. Lin and Y. Jia, "Consensus of a class of second-order multi-agent systems with time-delay and jointly-connected topologies," IEEE Trans. Autom. Control, vol. 55, no. 3, pp. 778-784, Mar. 2010.

[45] W. Tang, G. Zhong, G. Chen, and K. Man, "Generation of $n$-scroll attractors via sine function," IEEE Trans. Circuits Syst. I, Fundam. Theory Appl., vol. 48, no. 11, pp. 1369-1372, Nov. 2001.

[46] G. Jiang and W. Tang, "A global synchronization criterion for coupled chaotic systems via unidirectional linear error feedback approach," Int. J. Bifurcation Chaos, vol. 12, no. 10, pp. 2239-2253, 2002.

[47] D. J. Hill and G. Chen, "Power systems as dynamical networks," in Proc. IEEE Int. Symp. Circuits Syst., 2006, pp. 722-725.

[48] P. Kundur, J. Paserba, V. Ajjarapu, G. Anderson, A. Bose, C. Canizares, and N. Hatziargyriou et al., "Definition and classification of power system stability," IEEE Trans. Power Syst., vol. 19, no. 2, pp. 1387-1401, May 2004.

[49] A. Jadbabaie, J. Lin, and S. A. Morse, "Coordination of groups of mobile autonomous agents using nearest neighbor rules," IEEE Trans. Autom. Control, vol. 48, no. 6, pp. 988-1001, Jun. 2003.

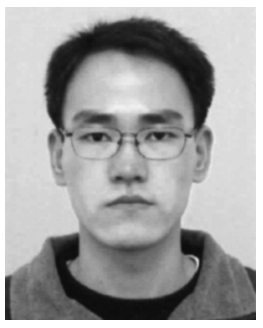

Tao Liu received the B.E. degree from Northeastern University, Shenyang, China, in 2003. He is currently working toward the Ph.D. degree in the School of Engineering at the Australian National University, Canberra, Australia.

His research interests include switched systems and dynamical networks.

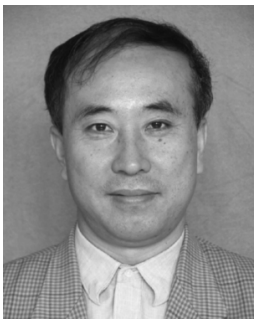

Jun Zhao was born in 1957. He received the B.S. and M.S. degrees in mathematics from Liaoning University, China, in 1982 and 1984, respectively and the Ph.D. degree in control theory and applications at Northeastern University, China, in 1991.

From 1992 to 1993, he was a Postdoctoral Fellow at Northeastern University, China. Since 1994 he has been with the School of Information Science and Engineering, Northeastern University, China, where he is currently a Professor. From February 1998 to February 1999, he was a Senior Visiting Scholar at the Coordinated Science Laboratory, University of Illinois at Urbana-Champaign, From November 2003 to May 2005, he was a Research Fellow at Department of Electronic Engineering, City University of Hong Kong. Since November 2006, as a Fellow, he has been with School of Engineering, the Australian National University, Canberra, Australia. His main research interests include switched systems, hybrid control, nonlinear systems, and robust control.

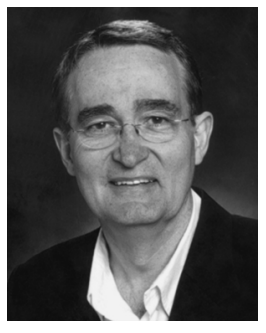

David J. Hill (M'76-SM'91-F'93) received the B.E. degree in electrical engineering and the B.Sc. degree in mathematics from the University of Queensland, Australia, in 1972 and 1974, respectively, and the $\mathrm{Ph} . \mathrm{D}$. degree in electrical engineering from the University of Newcastle, Australia, in 1976.

$\mathrm{He}$ is currently a Professor in the School of Engineering at the Australian National University (ANU) and Principal Researcher in the NICTA Canberra Research Laboratory. He is also a Chief Investigator of the Australian Research Council Centre of Excellence for Mathematics and Statistics of Complex Systems. During 2005-2010, he was an Australian Research Council Federation Fellow at ANU. He held academic and substantial visiting positions at the universities of Melbourne, California (Berkeley), Newcastle (Australia), Lund (Sweden), Sydney (Australia), and Hong Kong (City University). He currently holds honorary professorships at the University of Sydney, City University of Hong Kong, South China University of Technology, Wuhan University, and Northeastern University, China. His research projects are in network systems, stability analysis, nonlinear and distributed control and applications to infrastructure type networks, mainly smart grids.

Prof. Hill is a Fellow of the Institution of Engineers, Australia, the Society for Industrial and Applied Mathematics, and the Australian Academy of Science. $\mathrm{He}$ is also a Foreign Member of the Royal Swedish Academy of Engineering Sciences. 